\title{
Structure Optimization of a Vibration Suppression Device for Underwater Moored Platforms Using CFD and Neural Network
}

\author{
Zhaoyong Mao and Fuliang Zhao \\ School of Marine Science and Technology, Northwestern Polytechnical University, Xian 710072, China \\ Correspondence should be addressed to Zhaoyong Mao; maozhaoyong@nwpu.edu.cn
}

Received 8 July 2017; Accepted 13 September 2017; Published 11 December 2017

Academic Editor: Junpei Zhong

Copyright (c) 2017 Zhaoyong Mao and Fuliang Zhao. This is an open access article distributed under the Creative Commons Attribution License, which permits unrestricted use, distribution, and reproduction in any medium, provided the original work is properly cited.

\begin{abstract}
We only consider the underwater mooring platform (UMP) and the plate moving in the transverse direction, and the plate can be relative to the UMP free rotation. In the case of constant flow rate $(U=1 \mathrm{~m} / \mathrm{s})$, the effect of different dimensionless plate length $\left(L_{p} / D\right)$ and damping value $(c)$ on the UMP was studied. We get the sample data point set by computational fluid dynamics (CFD) simulation with changing the dimensionless plate length $\left(L_{p} / D=0.3,0.5,0.75,1.0,1.25,1.5\right)$ and damping value $(c=50$, $75,100,125,175,250,300(\mathrm{~N} \times \mathrm{s} / \mathrm{m}))$. The optimal value of the vibration suppression rate is obtained by backpropagation (BP) neural network and genetic algorithm. The optimal vibration suppression rate is $P_{y}=0.9878$ and the corresponding variable value is $L_{p} / D=1.0342, c=57.9631(\mathrm{~N} \times \mathrm{s} / \mathrm{m})$. In order to verify the accuracy of the optimization, we perform the CFD numerical simulation with the optimized parameters and compare the theoretical optimization results with the CFD simulation result. The absolute error between CFD simulation and optimal $P_{y}$ is only 0.0037 . Finally, we compare the results of CFD simulation based on optimal parameter with the bare UMP and analyze their dimensionless amplitude, wake structure, and lift coefficient. It is shown that BP neural network and generic algorithm are effective.
\end{abstract}

\section{Introduction}

The underwater mooring platforms (UMPs) are a class of underwater devices and with an anchor link to work in the sea. UMPs have broad application in both civil and military missions. Pressure, water quality detection, camera and acoustic communication, and other sensors are installed on the platform. It can be used for marine environmental management, resource protection, disaster monitoring, and marine hydrological monitoring in civil areas, and it also can be used for our army surface ships and submarines for remote reconnaissance defense. UMPs require as much as possible to stabilize and impact resistance to ensure that a variety of sensors can work properly. Therefore, in order to improve the stability of the UMP, it is very meaningful to study the vibration suppression control technology of UMP. At present, the research on the characteristics of the UMP is mainly focused on the simulation of the anchor chain and the cable, while the research on the stability of the UMP is especially less. Research on the underwater vibration control is mainly concentrated in the field of offshore riser.

The suppressing method of vortex-induced vibration can be divided into two types: active control and passive control measures. Active control measures are real-time monitoring of flow field changes and structural forces. It is through the automatic technology to disturb the flow filed, which can control the cylinder vortex shedding and finally achieve the purpose of vibration suppression. Passive control measures are to change the flow field by directly modifying the external shape of the structure or other devices attached to the structure. It is to achieve the purpose of controlling the formation and shedding of the vortex. Compared with the active control measures, passive control measures are simple to design, easy to manufacture, and low cost. So it has been widely used in the field of marine engineering.

In this paper, taking into account the stability of the UMP, we have adopted a relatively simple vibration suppression 
device-splitter plate which can rotate freely relative to the UMP.

In the previous research literatures, there are many studies of the cylinder with the fixed splitter plate and less research on the splitter plate that is free to rotate behind the cylinder. The majority is to study the effect of fixed dividers on vortex shedding. For the elastic-supported cylinder-splitter plate system, Ding et al. [1] studied the wake characteristics and vortex-induced vibration characteristic of the splitter plate under high Reynolds number, where the length of the splitter plate is the same as the diameter of the cylinder. The results show that, with high Reynolds number, the amplitude of the cylinder with plate dose not decrease (without the effect of suppressing vibration) but increases as the number of Reynolds increases. The vibration frequency is lower than the bare cylinder and the vortex shedding model of cylinder with a splitter plate and a bare cylinder is also different. The inhibitory effect is more obvious as the velocity decreases. Tan et al. [2] carried out a two-dimensional numerical simulation with a splitter plate cylinder and a bare cylinder, and the ratio of the length of the splitter plate to the diameter of the cylinder varies from 0.25 to $2.0(L / D=0.25 \sim 2.0)$. The results show that the splitter plate has the most significant effect on the vortex-induced vibration control of the riser when $L / D$ is 1.0-2.0. Wang et al. [3] performed a two-dimensional computational fluid dynamics (CFD) numerical simulation of the fixed separation plate (variable dimensionless length). When the Reynolds numbers are 1000 and 30000, respectively, changing $L / D=0.5 \sim 2.0$ to simulation can be found to reduce the drag coefficient, lift coefficient, and vortex shedding frequency. This document of Amiraslanpour et al. [4] is used to study the two splitter plate in the upstream or downstream stages of the cylinder. They found the influence of the splitter plate on the drag force of the cylinder. They also studied the effect of the distance from the end of the splitter to the cylindrical surface without much impact on the resistance. When the ratio of amplitude to the cylinder diameter $(A / D)$ was 0.25 and 0.5 , respectively, the resistance was reduced by $57 \%$ and $36 \%$. Qiu et al. [5] experimentally studied the fixed cylinder with frontal plate, bare cylinder, cylinder with rear splitter plate, cylinder with bilateral plates, and semicylindrical roof. They found that the cylinder with wake splitter plate $(L / D=3)$ can effectively inhibit the vortex shedding, and shear layer was separated on both sides of the plate. Lou et al. [6] experimentally studied the effect of vortex-induced vibration of two adjacent risers with splitter plates and found that the splitter plate on the risers was effectively in suppressing both cross-flow (CF) and in-line (IL). Assi et al. [7] experimented with plain cylinder, cylinder with fixed splitter plate, cylinder with free-to-rotate splitter plate, and cylinder with pairs of plates and studied their vibration amplitude at different reduced speed. The results show that these devices can effectively reduce the resistance and suppress vortex vibration. Assi et al. [8] have mainly studied the stability of a free-to-rotate short-tail fairing and a splitter plate as suppressors of vortex-induced vibration. They studied the rotating (free-to-rotate suppressors in 2dof) and nonrotating (fixed suppressors in 1-dof) at different reduced speed. It is found that the free-rotating suppressors will be deflected position to remain stable and extend the vortex shedding and suppressing vortex-induced vibration. It is also found that fixed suppressors will show serious gallop in a considerable flow rang of flow speeds. Gozmen et al. [9] and Zhu et al. [10] studied the relationship of different lengths of splitter plate to suppress vortex shedding. Gu et al. [11] studied a fixed cylinder with a free splitter plate, where the ratio of the length of plate to the diameter of the cylinder varied from 0.5 to 6 and the Reynolds number varied from 30000 to 60000. The pressure distribution, drag, lift force, and vortex shedding model are analyzed, and the study finds that the free rotation angle corresponding to the length of the longer plate is smaller. Akilli et al. [12] studied that the influence of factors such as the dimensionless thickness $(T / D)$ on the splitter plate and the distance $(G / D)$ between the end of the plate and the fixed cylinder vortex shedding was studied. The dimensionless thickness of the plate varies from 0.016 to 0.08 , and the distance is changed from 0 to $100(\mathrm{~mm})$ in the step size of 12.5 . When $G / D$ changed from 0 to 1.75 , the plate has effect on suppression of the vortex shedding. Law and Jaiman [13] studied four kinds of vibration-proofing devices (fairing, connected-C, disconnected-C, and splitter plate) separately, which the device can not rotate freely relative to the cylinder. It was found that the connected- $\mathrm{C}$ device was similar to the effect of the fairing device on vortex-induced vibration. The study also found that the connected- $\mathrm{C}$ device and disconnected- $\mathrm{C}$ device can effectively prevent the cylinder galloping at high reduced velocity, but the connection between them has little effect on vibration suppression. In [14], the authors mainly studied the circular cylinders fitted with three different geometries of splatter plate (solid splitter plate $L / D=0.5$, solid splitter plate $L / D=1.0$, and slotted splitter plate $L / D=1.0)$. He studied the response of the different vibration mechanisms of the three devices with varying reduced speeds.

With reference to the above-mentioned latest literature, there are few studies on the splitter plate parameters and the splitter plate which are rotated relative to the cylinder. So in this paper, I mainly studied the effect of the length of the splitter plate that can rotate freely around the cylinder and the damping values between the splitter plate and the cylinder on the vibration suppression technique of the UMP.

For some complex numerical simulation, if we all use CFD simulation solution, then we will greatly increase the calculation of cost and the calculation cycle. Therefore, in order to improve the computational efficiency, this paper establishes an optimization method based on the combination of CFD and backpropagation (BP) neural network and genetic algorithm. BP neural network and genetic algorithm are used at some of the nonlinear data to find the optimal value [15-17]. Adaptive control [18, 19] in the neural network has also been a very good application. Safikhani et al. [20] have studied the multiobjective optimization of nanofluid flow in flat tubs, combined with a CFD, artificial neural networks, and genetic algorithms. They got important design information about nanofluids and flat tubes by combining CFD, GMDH, and THE multiobjective optimization method. Avci et al. [21] studied the optimization of the deign parameters of a home refrigerators using CFD and artificial neural 


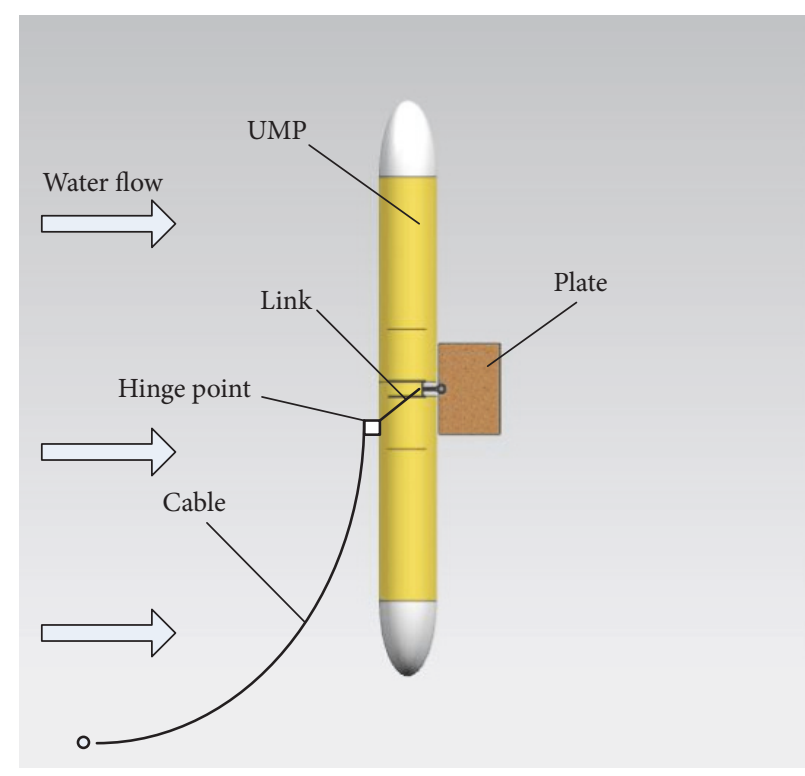

(a)

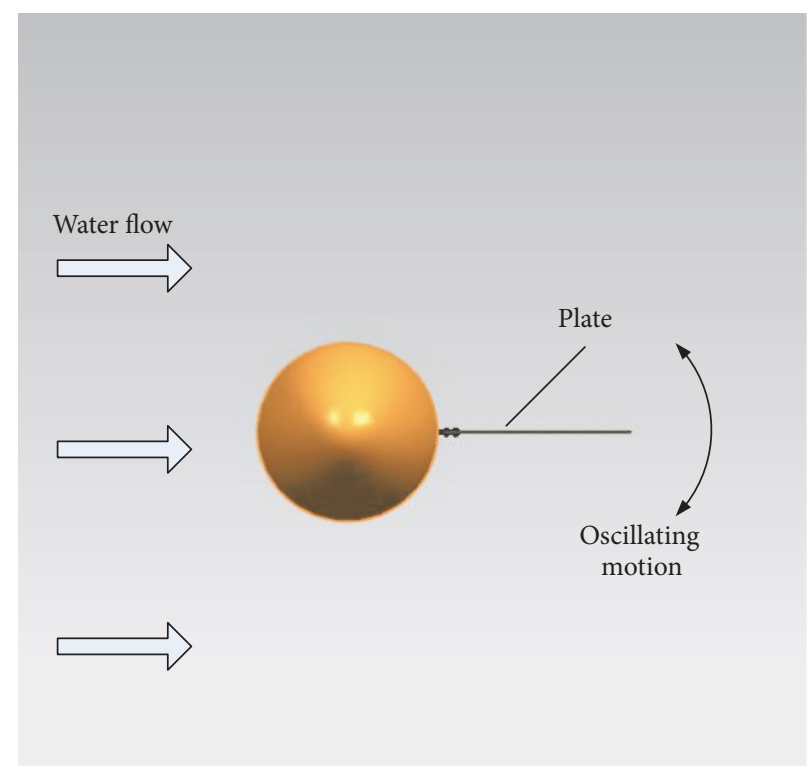

(b)

FIgURE 1: (a) Overall diagram of the UMP. (b) A schematic diagram of the splitter plate for rotation.

network. This paper shows that CFD simulation and the ANN can determine the best value for the refrigerator design.

So we can combine CFD simulation and BP neural network genetic algorithm to optimize the best vibration suppression rate. Through the use of CFD simulation software, we can get some data about vibration suppression rate, finally combined with BP neural network genetic algorithm to find the best suppression effect and the corresponding dimensionless plate length and damping value size.

\section{Geometry Configuration}

One end of the cable is tethered on the UMP and the other end is fixed under the sea, so that the UMP is floating in the sea. Some sensor can not work properly, because the ocean flow easily leads to the vortex-induced vibration. Inspired from the above research, we installed a splitter plate in the wake area of the UMP, in which the splitter plate can freely rotate around the UMP. As shown in Figure 1, (a) is the overall diagram of the UMP, and (b) is a schematic diagram of the splitter plate for rotation. The vortex shedding process alternates on both sides of the UMP and will produce vortex-induced vibrations because of the UMP generated periodic pulsating forces. So, we designed a rotatable splitter plate to extend the process and achieve the effect of vibration suppression. In this paper, we mainly focus on evaluating the wake structure, force, motion (the UMP only moves in the transverse direction with a degree of freedom, and the splitter plate is rotated relative to UMP), and the transverse amplitude at different design parameters with a CFD method.

Because the two-dimensional model requires less grid and shorter calculation time, the general vortex-induced vibration numerical simulation is based on two-dimensional numerical simulation. A simplified two-dimensional (2D)

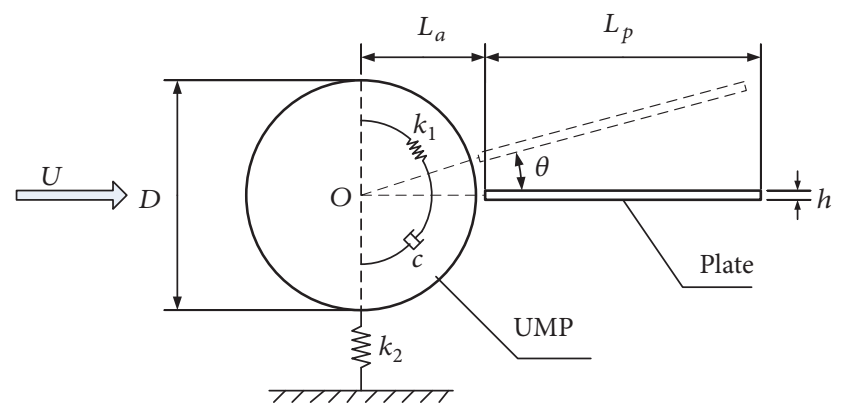

Figure 2: A simplified 2D model of UMP with a splitter plate.

model of UMP with a splitter plate is shown in Figure 2. The parameters indicated in Figure 2 that $U$ represents the velocity of the upstream flow; $L_{p}$ represents the length of the plate; $L_{a}$ represents the distance between the root of the plate and the center of the UMP; $\theta$ represents the rotation angle of the plate; $k_{1}$ represents the spring stiffness between the plate and the UMP; $c$ represents system damping; $D$ represents diameter of the UMP and $h$ represents the height of the plate; $k_{2}$ represents the simplified spring stiffness between the UMP and the cable. Where $k_{2}$ is derived from $(1), k_{2}=1599(\mathrm{~N} / \mathrm{m})$ value is the constant value in this paper.

$$
\begin{aligned}
F d \cdot l \cdot \sin (\alpha) & =k_{2} \cdot \alpha \\
k_{2} & =F d \cdot l .
\end{aligned}
$$

In this paper, we set the distance between the center of the UMP and the root of plate $L_{a}=0.3(\mathrm{~m})$ and the diameter of the cylinder is $D=0.533(\mathrm{~m})$. A typical flow velocity of $U=1 \mathrm{~m} / \mathrm{s}$ is selected for all simulations in this paper.

The specific values of each parameter are shown in Table 1. 
TABLE 1: Parameters in simulation.

\begin{tabular}{lcccccccc}
\hline Parameter & $D$ & $U$ & $L_{a}$ & $L_{p}$ & $k_{1}$ & $k_{2}$ & $h$ & $c$ \\
\hline Value & $0.533 \mathrm{~m}$ & $1 \mathrm{~m} / \mathrm{s}$ & $0.3 \mathrm{~m}$ & $\sim$ & $\sim$ & $1599 \mathrm{~N} / \mathrm{m}$ & $0.002 \mathrm{~m}$ & $\sim$ \\
\hline
\end{tabular}

Note. " $\sim$ " indicates that the value is variable.

2.1. Governing Equations and Turbulence Model. In this paper, the numerical simulation of the calculation medium is incompressible viscous fluid and does not take into account the temperature changes. So it belongs to the unsteady flow. The governing equations include continuity equations and momentum equations, as shown as follows:

$$
\begin{aligned}
& \frac{\partial u_{x}}{\partial x}+\frac{\partial u_{y}}{\partial y}=0 \\
& \frac{\partial u_{x}}{\partial t}+u_{x} \frac{\partial u_{x}}{\partial x}+u_{y} \frac{\partial u_{x}}{\partial y}=-\frac{1}{\rho} \frac{\partial p}{\partial x}+v\left(\frac{\partial^{2} u_{x}}{\partial x^{2}}+\frac{\partial^{2} u_{x}}{\partial y^{2}}\right) \\
& \frac{\partial u_{y}}{\partial t}+u_{x} \frac{\partial u_{y}}{\partial x}+u_{y} \frac{\partial u_{y}}{\partial y} \\
& =-\frac{1}{\rho} \frac{\partial p}{\partial y}+v\left(\frac{\partial^{2} u_{y}}{\partial x^{2}}+\frac{\partial^{2} u_{y}}{\partial y^{2}}\right),
\end{aligned}
$$

where $u$ represents the velocity of the fluid in the $i$ direction; $\rho$ represents the fluid density; $v$ represents the kinematic viscosity coefficient of the fluid; $i, j$ represent the direction of the different axes.

At present, the turbulence model commonly used in computational fluid dynamics mainly includes Reynolds stress model and viscous vortex model. The Reynolds stress model is a direct complement to the equation that expresses the Reynolds stress equation to solve the time-averaged motion control equation. The vortex model is the assumption that the turbulence viscosity and the vorticity coefficient are introduced to supplement the Reynolds stress tensor $-\rho \overline{u_{i} u_{j}}$ and (3) can be seen.

$$
-\rho \overline{u_{i} u_{j}}=\mu t\left(\frac{\partial U_{i}}{\partial x_{j}}+\frac{\partial U_{j}}{\partial x_{i}}\right)-\frac{2}{3}\left(\rho k+\mu \frac{\partial U_{i}}{\partial x_{i}}\right) \delta_{i j},
$$

where $\mu_{t}$ represents turbulent vortex viscosity coefficient; $k$ represents turbulent kinetic energy, in which

$$
k=\frac{1}{2} \overline{u_{i} u_{j}}=\frac{1}{2}\left(\overline{u_{x}^{\prime 2}}+\overline{u_{y}^{\prime 2}}+\overline{u_{z}^{\prime 2}}\right) .
$$

The SST $k-\omega$ model combines the advantages of the $k-\varepsilon$ model and the $k$ - $\omega$ model, namely, the advantages of the boundary layer problem and the superior field calculation. Using the SST $k$ - $\omega$ turbulence model to numerically simulate the vortex-induced vibration problem is more accurate, so this paper uses the SST $k$ - $\omega$ model to carry out numerical simulation.

2.2. Equations of Motion. The dynamic equation of the plate which can freely rotate around the UMP can be obtained by a typical mass-spring-damper system and be presented as

$$
J \ddot{\theta}(t)+c \dot{\theta}(t)+k_{1} \theta(t)=M(t),
$$

where $J$ is the total rotational inertial of the plate; $c$ is the system damping, $k_{1}$ is the stiffness of the spring, $\theta$ is the oscillating angle of the plate, and $M(t)$ is the hydrodynamic force on the plate.

The total rotational inertial $(J)$ is the sum of the plate structure rotational inertial $\left(J_{b}\right)$ and the added mass component $\left(J_{a}\right) . J_{b}$ and $J_{a}$ can be calculated by following equation, respectively:

$$
\begin{aligned}
J & =J_{a}+J_{b} \\
J_{b} & =\frac{1}{3} \rho_{\text {plate }} h L_{\text {feature }}\left[\left(L_{a}+L_{p}\right)^{3}-L_{a}{ }^{3}\right] \\
J_{a} & =\frac{1}{16} \pi \rho_{\text {water }} L_{\text {feature }}\left[\left(L_{a}+L_{p}\right)^{4}-L_{a}{ }^{4}\right],
\end{aligned}
$$

where $L_{\text {feature }}$ is the feature length of the UMP and plate, $L_{\text {feature }}=1 \mathrm{~m} ; \rho_{\text {plate }}$ is the density of the plate; $h$ is the height of the plate; $L_{a}$ represents the distance between the root of the plate and the center of the UMP; $L_{p}$ represents the length of the plate; $\rho_{\text {water }}$ is the density of the water.

The vibration equation (1-dof) of the UMP only in the transverse direction can be obtained by the mass-spring stiffness system and be presented as

$$
M \ddot{y}(t)+k_{2} y(t)=F_{l}(t),
$$

where $M$ is the total mass of the UMP which is sum of the total mass of the UMP $\left(M_{1}\right)$ and the total mass of the plate $\left(M_{2}\right)$. The above $M_{1}$ is the sum of the additional mass of the UMP $\left(M_{\text {add(UMP) }}\right)$ and the mass of the UMP itself $\left(M_{\text {itself(UMP) }}\right) ; M_{2}$ is the sum of the additional mass of the plate $\left(M_{\text {add(Plate) }}\right)$ and the mass of the plate itself $\left(M_{\text {itself(Plate) }}\right)$. Since the angle of the freely rotating plate is small, the additional mass of the plate $\left(M_{\text {add(Plate) }}\right)$ is approximately calculated as a cylinder with a diameter equal to its length. As shown in formula (10). $k_{2}$ is the equivalent stiffness between the cable and the UMP, which can be obtained from formula (1). $F_{l}$ is the total lift of the whole system that is the sum of the cylinder lift of the cylinder lift and plate lift. $y, \dot{y}$, and $\ddot{y}$ are the displacement, velocity, and acceleration in the $y$ direction (transverse vibration direction).

$$
\begin{aligned}
M & =M_{1}+M_{2} \\
M_{1} & =M_{\text {add(UMP) }}+M_{\text {itself(UMP) }} \\
M_{\text {itself(UMP) }} & =200 \mathrm{~kg} \\
M_{\text {add(UMP) }} & =\pi \times\left(\frac{D}{2}\right)^{2} \times L_{\text {feature }} \times \rho_{\text {water }} \times C_{p} \\
M_{2} & =M_{\text {add(plate })}+M_{\text {itself(plate })} \\
M_{\text {itself(plate) }} & =L_{p} \times h \times L_{\text {feature }} \times \rho_{A l} \\
M_{\text {add(plate })} & =\pi \times\left(\frac{L_{p}}{2}\right)^{2} \times L_{\text {feature }} \times \rho_{\text {water }} \times C_{p},
\end{aligned}
$$

where $L_{\text {feature }}$ is the feature length of the UMP and plate, $L_{\text {feature }}=1 \mathrm{~m} ; \rho_{\text {water }}$ is density of the water, $\rho_{\text {water }}=$ 


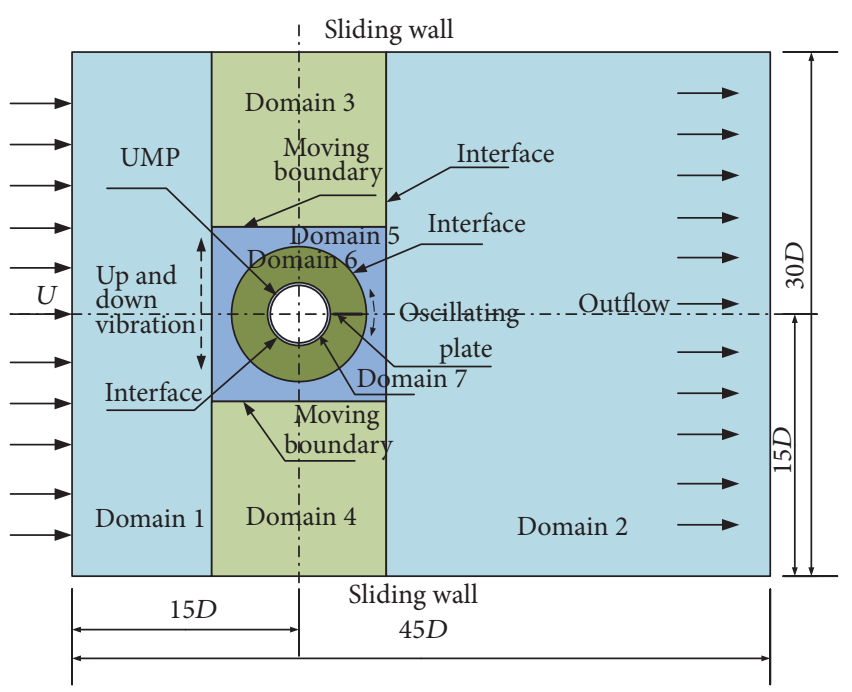

FIGURE 3: Computational domain and boundary conditions.

$1000 \mathrm{~kg} / \mathrm{m}^{3} ; C_{p}$ is an additional mass factor, $C_{p}=1 ; \rho_{A l}$ is the density of the plate, $\rho_{A l}=2700 \mathrm{~kg} / \mathrm{m}^{3}$.

In order to eliminate the dimensional effects, the lift, drag, and torque are also normalized, and the natural frequency of the plate is shown in (14).

$$
\begin{aligned}
C_{l} & =\frac{F_{l}}{(1 / 2) \rho_{\text {water }} D H U^{2}} \\
C_{d} & =\frac{F_{d}}{(1 / 2) \rho_{\text {water }} D H U^{2}} \\
C_{m} & =\frac{M}{(1 / 4) \rho_{\text {water }} D^{2} H U^{2}} \\
f & =\frac{1}{2 \pi} \sqrt{\frac{k_{1}}{J} .}
\end{aligned}
$$

\section{Numerical Approach}

3.1. Computational Domain and Boundary Conditions. A rectangle with a length of $45 \mathrm{D}$ and a width of $30 \mathrm{D}$ is used as the computational domain of this paper. As shown in Figure 3, it can be seen from the figure that the center of UMP is placed at a distance from the left boundary of the computational domain to $15 \mathrm{D}$ and on the symmetry axis of the of the top and bottom boundary. The overall domain is split into seven subdomains, including two external static domains, two dynamic domains for updating the grid with layering method [22, 23], two inner stationary domains 5 and 6 , and an oscillating domain. This inner stationary domain 7 contains the cells around the UMP. The rotation domain where the plate is located is divided by two sliding interfaces in two inner stationary domains (domain 5 and domain 7). The stationary domain 5 has two upper and lower moving boundaries for the grid update. The grid updating domain (domain 3 and domain 4) and the inner entire UMP motion domain (domain 5, domain 6, and domain 7) are divided

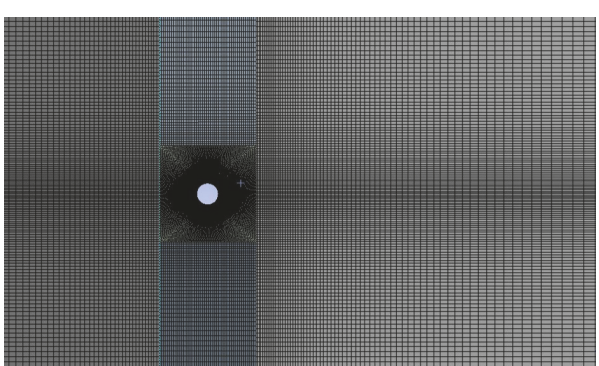

FIGURE 4: The mesh graph for the entire computational domains.

by an external stationary domain (domain 1 and domain 2). The following is a description of the boundary conditions: (1) inlet: set the uniform and steady flow of incoming speed $1 \mathrm{~m} / \mathrm{s}$; the corresponding Reynolds number is 533000; (2) outlet: outflow exit boundary conditions; (3) interface: the overlapped edges between the seven domains are set as interfaces; (4) wall: the surface of the UMP and the plate are set to no slip boundary conditions, whereas the top and the bottom edges are set to slip boundary conditions.

3.2. Mesh Generation. The entire computational domains are a quadrilateral mesh cell. It is worth nothing here that the rigid boundary layer must be added to the surface of the UMP and plate which the boundary layer grid moves with the UMP and plate and is stable and the shape no longer changes to provide sufficient precision. The first layer height of the boundary layer is determined according to the value of $y^{+}$ which is suggested by Fluent User's Guide [24] for the use of the SST $k$ - $\omega$ turbulence model. So here set the first layer height as $0.0002 \mathrm{~m}$, and UMP and plate boundary layer were set to 16 layers and 12 layers. Figure 4 shows the mesh graph for the entire computational domains. Figure 5 shows a mesh diagram of UMP and plate. The oscillating domain rotates about the center of the UMP and rotates along the interfaces, and the mesh of the rotation domain does not change and update.

\subsection{Mesh Independence, Numerical Method, and Time Step Validation}

3.3.1. Mesh Independence Validation and Numerical Method Validation. Using the CFD numerical simulation, the meshing and time step have a significant effect on the results. A mesh independence verification study is conducted using three grids with different nodes densities (41000/66900/ 97000 elements). The mesh verification is carried out by means of a fixed cylinder and not rotating the plate $(k 1=\infty$, $k 2=\infty, c=\infty$, and $L p=D)$. The independence of the mesh is verified by assessing the root mean square (RMS) of the lift coefficient and the mean drag coefficient for different mesh densities. The results are summarized in Table 2. Both $C_{l}$ and $C_{d}$ have the good consistency for different mesh densities. Accordingly, the mesh with medium density can predict the performance with sufficient accuracy and will be used in the later simulations. 

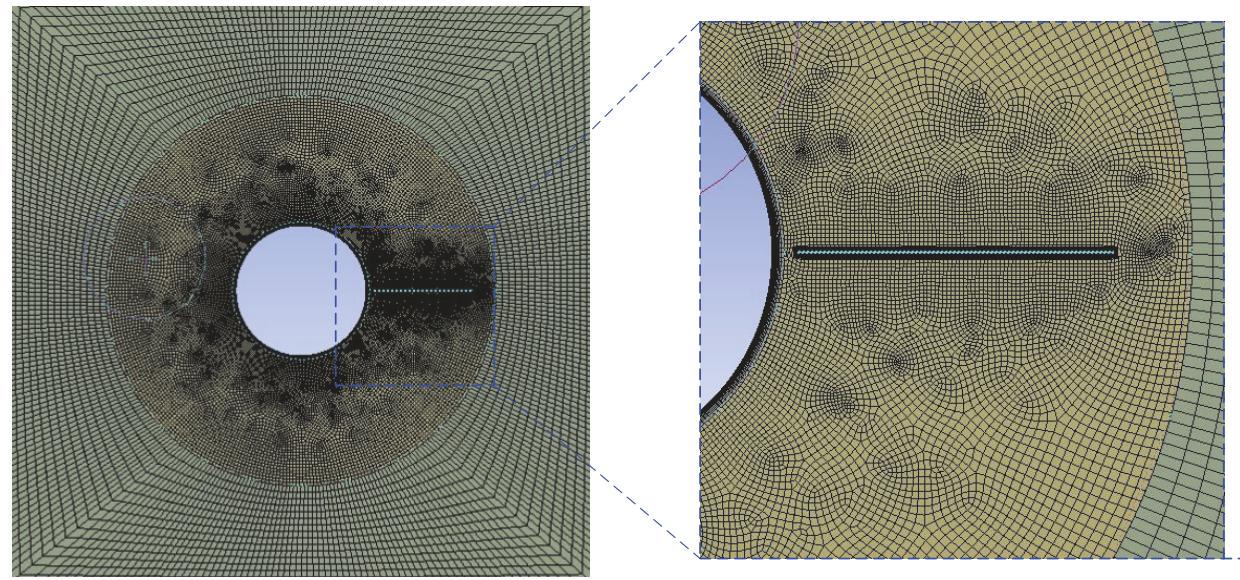

FIgURE 5: Mesh diagram of UMP and plate.

TABLE 2: Mesh convergence results.

\begin{tabular}{lccc}
\hline Mesh & Number of elements & RMS of Cl & Averaged Cd \\
\hline$(1)$ & 41000 & 0.145321 & 0.515543 \\
$(2)$ & 66900 & 0.162324 & 0.571632 \\
$(3)$ & 97000 & 0.172251 & 0.583214 \\
\hline
\end{tabular}

TABLE 3: Physical model parameters for the simulation.

\begin{tabular}{lcc}
\hline Description & Symbol & Value \\
\hline Mass ratio & $m^{*}$ & 0.93 \\
Damping ratio & $\xi_{\text {total }}$ & 0.1076 \\
Velocity ratio & $U^{*}$ & $3-13$ \\
Spring stiffness per unit length & $K(\mathrm{~N} / \mathrm{m})$ & 76.5 \\
Diameter & $D(\mathrm{~mm})$ & 32 \\
\hline
\end{tabular}

Some of the previous experiments have been done on the study of the splitter plate in the wake of a cylinder. In order to prove the validity of the numerical method, we use CFD to simulate a 1-degree-of-freedom vortex-induced vibration with a cylinder and then compare the numerical results with the experimental data. The testing parameters adopted in the validation simulations are the same with the experiments and are listed in Table 3.

Figure 6 shows the comparison of the amplitude and frequency response between the numerical and the experimental results. It is observed that the VIV amplitudes obtained from the numerical method are in good agreement with the experimental data and the lock-in of amplitude (corresponding to the upper branch) is well characterized, except a slightly overestimate at high flow velocities. As for the frequency, the numerical results coincident with the experimental results well. Therefore, comparison with experiments shows that the numerical method is quite credible and acceptable.

3.3.2. Time Step Validation. In this paper, the numerical simulation parameters of the time step verification are the same as the simulation parameters of this paper. We just
TABLE 4: Different time steps of the RMS of lift and averaged drag force.

\begin{tabular}{lcc}
\hline Time step & RMS of $\mathrm{Cl}$ & Averaged Cd \\
\hline 0.001 & 0.169321 & 0.574532 \\
0.002 & 0.168376 & 0.573810 \\
0.005 & 0.162324 & 0.571632 \\
0.01 & 0.150719 & 0.567762 \\
0.02 & 0.127477 & 0.559646 \\
\hline
\end{tabular}

simulate the flow of the fixed cylinder with a plate to verify the time step. We carry out the numerical simulation with the dimensionless plate length 1 . The time step is $0.001,0.002$, $0.005,0.01$, and 0.02 . We validate the effective time step by referring to the root mean square (RMS) and the mean drag coefficient at different time steps. This will not only get accurate results but also improve the efficiency of computing. The results are summarized in Table 4. The lift coefficient curve is shown in Figure 7. Table 4 and Figure 7 show that, in the premise of ensuring the accuracy of the calculation, while improving the efficiency of the calculation, we choose 0.005 as the time step of this paper.

\section{Numerical Simulation}

4.1. Numerical Simulation Conditions. In the whole numerical simulation of this paper, we mainly study the effects of plate length and damping (damping between UMP and plate) variation on the UMP amplitude. In addition, the thickness of the plate and the front of the plate to the center of the UMP $\left(L_{a}\right)$ also have a certain impact on the vibration suppression rate. In order to reduce the amount of calculation, we set the plate thickness and distance $\left(L_{a}\right)$ as a constant. Other related numerical simulation parameters are $f(f=0.5$, natural frequency of the plate), $h$ ( $h=0.002 \mathrm{~m}$, height of the plate), and $k_{2}=1599(\mathrm{~N} \times \mathrm{s} / \mathrm{m})$ (as can be seen from (1)). Equations (6), (7), (8), and (14) show that when we change the plate length, the total moment of inertia of the plate also changes with (6), (7), and (8). The spring stiffness of the plate changes 

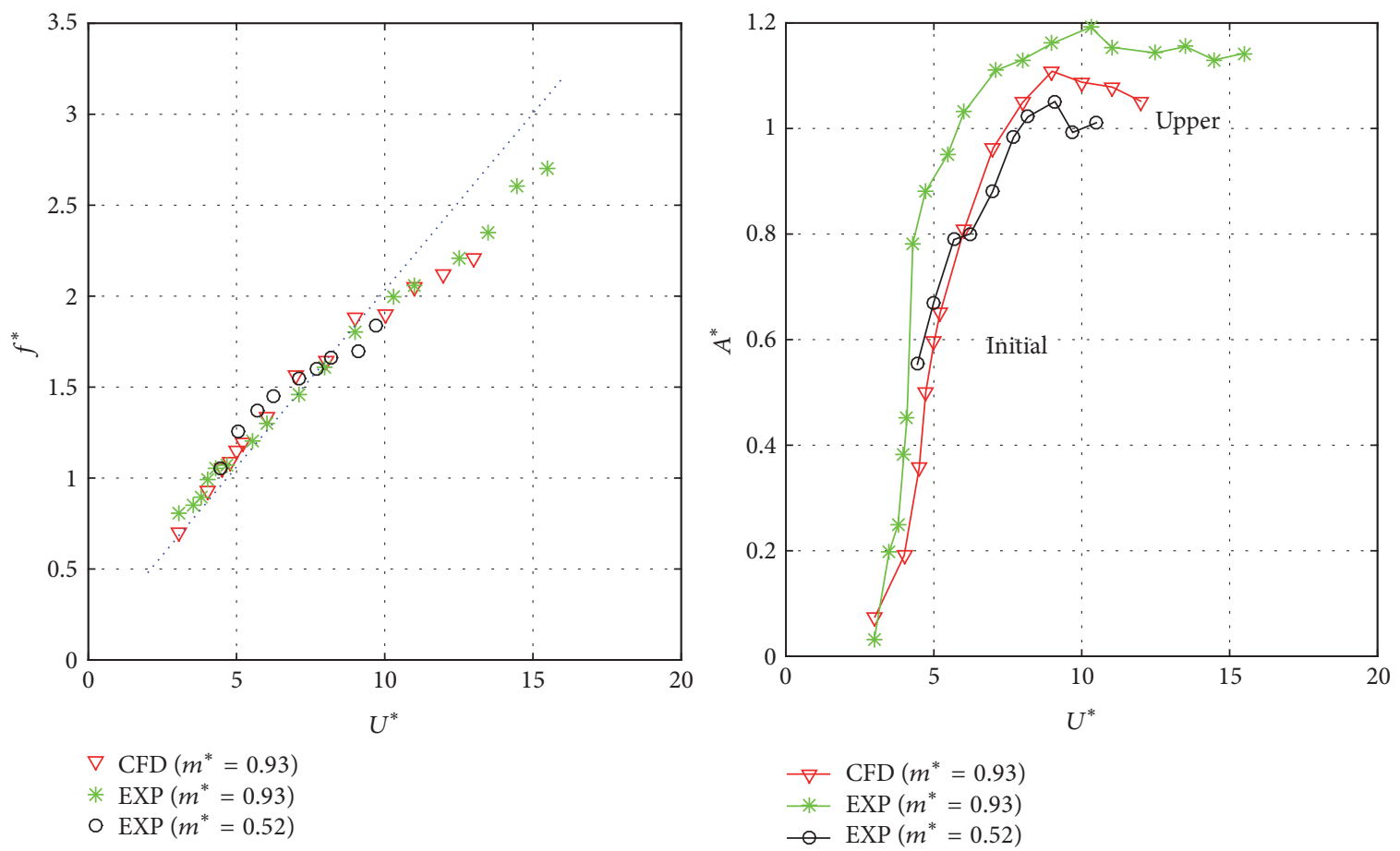

Figure 6: Comparison of amplitude and frequency responses between the numerical results and the experimental results.

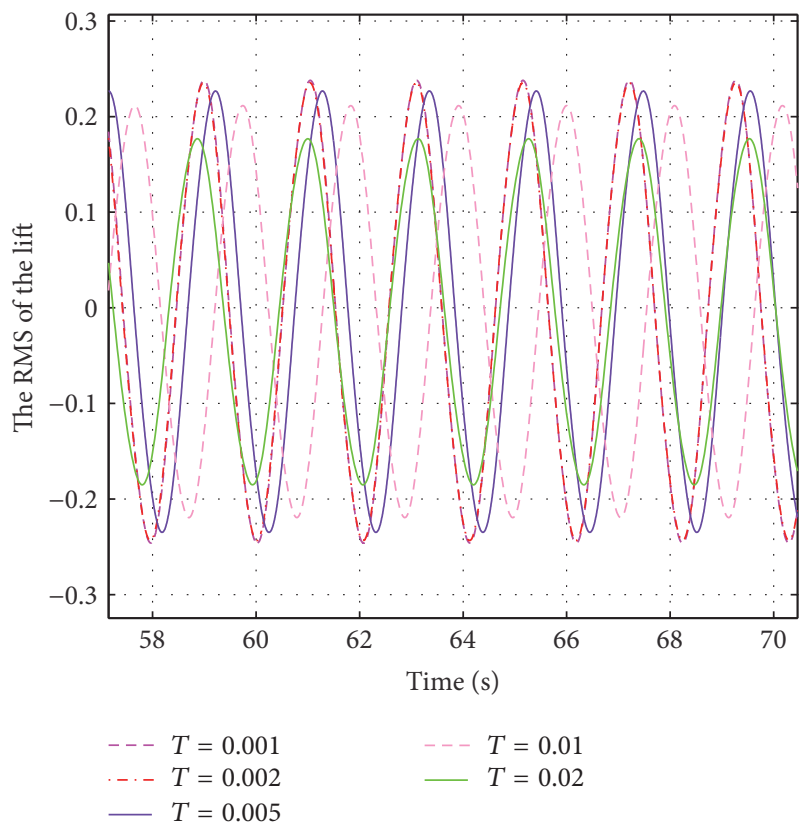

FIgURE 7: The lift coefficient of different time steps.

with the total moment of inertia of the plate is also obtained by (14). All case calculations, the density of the mesh, and time steps are all the same. Here we chose the plate length data $L_{p} / D=0.3,0.5,0.75,1.0,1.25$, and 1.5 and damping data 50 , $75,100,125,175,250$, and $300(\mathrm{~N} \times \mathrm{s} / \mathrm{m})$. The range of each variable is shown in Table 5 .
TABLE 5: Design variables and their range of variations.

\begin{tabular}{lccc}
\hline Design variables & From & To & Selected \\
\hline$L_{p} / D$ & 0.3 & 1.5 & $0.3,0.5,0.75,1.0,1.25,1.5$ \\
$c(\mathrm{~N} \times \mathrm{s} / \mathrm{m})$ & 50 & 300 & $50,75,100,125,175,250,300$ \\
\hline
\end{tabular}

4.2. Numerical Simulation Results. In this paper, the influence of dimensionless plate length $\left(L_{p} / D\right)$ and damping $(c)$ on the vibration of UMP is studied under the condition that the other parameters remain unchanged. We firstly simulate the vortex-induced vibration of a bare cylinder under the same computational simulation conditions. We compare the vibration amplitude, lift coefficient, and drag coefficient of the bare cylinder with the cylinder with the plate. The parameter of the study, the amplitude of the vibration $(A)$, the dimensionless amplitude $\left(A_{y}^{*}\right)$, and the suppression rate $P_{y}$ are shown in Table 6 . Here it is the dimensionless amplitude $A_{y}^{*}$ which is the ratio of the actual amplitude to the diameter of UMP.

Through Table 6, study found that when the dimensionless plate length $\left(L_{p} / D\right)$ is 0.3 and the damping value $(c)$ is greater than $75(\mathrm{~N} \times \mathrm{s} / \mathrm{m})$, the UMP does not show the effect of vibration suppression but increased the vibration amplitude.

From the finite discrete parameter data point, we can see that the amplitude of the UMP is the smallest when the dimensionless plate length $\left(L_{p} / D\right)$ is 1.25 , the damping $(c)$ is $175(\mathrm{~N} \times \mathrm{s} / \mathrm{m})$, and the maximum suppression rate $\left(P_{y}\right)$ is 0.979676 . The effect on the suppression vibration achieves the best results. 
TABLE 6: Numerical simulation parameter data display table.

\begin{tabular}{|c|c|c|c|c|c|}
\hline Number & $L_{p} / D$ & $c(\mathrm{~N} \times \mathrm{s} / \mathrm{m})$ & $A(\mathrm{~m})$ & $A_{y}^{*}$ & $P_{y}$ \\
\hline (1) & - & - & 0.451631 & 0.847339 & - \\
\hline (2) & 0.3 & 50 & 0.386184 & 0.724547 & 0.144914 \\
\hline (3) & 0.3 & 75 & 0.451842 & 0.847734 & -0.00047 \\
\hline (4) & 0.3 & 100 & 0.487460 & 0.914559 & -0.07933 \\
\hline (5) & 0.3 & 125 & 0.524511 & 0.984073 & -0.16137 \\
\hline (6) & 0.3 & 175 & 0.543321 & 1.019364 & -0.20302 \\
\hline (7) & 0.3 & 250 & 0.522656 & 0.980593 & -0.15726 \\
\hline (8) & 0.3 & 300 & 0.512464 & 0.961472 & -0.1347 \\
\hline (9) & 0.5 & 50 & 0.289810 & 0.543733 & 0.358304 \\
\hline (10) & 0.5 & 75 & 0.312187 & 0.585717 & 0.308757 \\
\hline (11) & 0.5 & 100 & 0.338961 & 0.635948 & 0.249475 \\
\hline (12) & 0.5 & 125 & 0.367375 & 0.689259 & 0.18656 \\
\hline (13) & 0.5 & 175 & 0.434383 & 0.814977 & 0.038191 \\
\hline (14) & 0.5 & 250 & 0.447285 & 0.839185 & 0.009622 \\
\hline (15) & 0.5 & 300 & 0.448789 & 0.842005 & 0.006293 \\
\hline (16) & 0.75 & 50 & 0.011683 & 0.021920 & 0.974131 \\
\hline (17) & 0.75 & 75 & 0.010332 & 0.019384 & 0.977124 \\
\hline (18) & 0.75 & 100 & 0.012791 & 0.023999 & 0.971677 \\
\hline (19) & 0.75 & 125 & 0.015718 & 0.029490 & 0.965197 \\
\hline (20) & 0.75 & 175 & 0.020008 & 0.037539 & 0.955698 \\
\hline (21) & 0.75 & 250 & 0.148309 & 0.278252 & 0.671616 \\
\hline (22) & 0.75 & 300 & 0.147588 & 0.276900 & 0.673212 \\
\hline (23) & 1.0 & 175 & 0.010143 & 0.019031 & 0.97754 \\
\hline (24) & 1.0 & 250 & 0.015051 & 0.028238 & 0.966674 \\
\hline (25) & 1.0 & 300 & 0.016659 & 0.031256 & 0.963113 \\
\hline (26) & 1.25 & 50 & 0.015277 & 0.028662 & 0.966174 \\
\hline (27) & 1.25 & 75 & 0.012806 & 0.024026 & 0.971645 \\
\hline (28) & 1.25 & 100 & 0.010937 & 0.020519 & 0.975784 \\
\hline (29) & 1.25 & 125 & 0.009723 & 0.018242 & 0.978471 \\
\hline (30) & 1.25 & 175 & 0.009179 & 0.017222 & 0.979676 \\
\hline (31) & 1.25 & 250 & 0.010298 & 0.019320 & 0.977199 \\
\hline (32) & 1.25 & 300 & 0.012559 & 0.023562 & 0.972192 \\
\hline (33) & 1.5 & 50 & 0.030614 & 0.057437 & 0.932215 \\
\hline (34) & 1.5 & 75 & 0.028877 & 0.054178 & 0.936061 \\
\hline (35) & 1.5 & 100 & 0.027484 & 0.051565 & 0.939145 \\
\hline (36) & 1.5 & 125 & 0.026268 & 0.049283 & 0.941838 \\
\hline (37) & 1.5 & 175 & 0.024477 & 0.045924 & 0.945802 \\
\hline (38) & 1.5 & 250 & 0.022894 & 0.042952 & 0.949309 \\
\hline (39) & 1.5 & 300 & 0.021951 & 0.041185 & 0.951395 \\
\hline
\end{tabular}

\section{Optimal Design Method}

For the unknown nonlinear function, it is difficult to accurately find the extremum of the function only through the input and output data of the function. This kind of problem can be solved by BP neural network and genetic algorithm. The nonlinear fitting ability of BP neural network and the nonlinear optimization ability of genetic algorithm are used to find the extreme value. For some complex numerical simulation, if we all use CFD simulation solution, then we will greatly increase the calculation of cost and the calculation cycle. Therefore, in order to improve the computational efficiency, this paper establishes an optimization method based on the combination of CFD and BP neural network and genetic algorithm. The optimization method is to obtain the sample point of the data by CFD simulation and then use BP neural network to establish the agent model between the influencing factors and the response value. Then the genetic algorithm is used to solve the optimization model, and the optimal problem is obtained solution.

5.1. BP Neural Network. BP neural network is a kind of multilayer feedforward network with error backpropagation, which has very strong nonlinear mapping approximation 


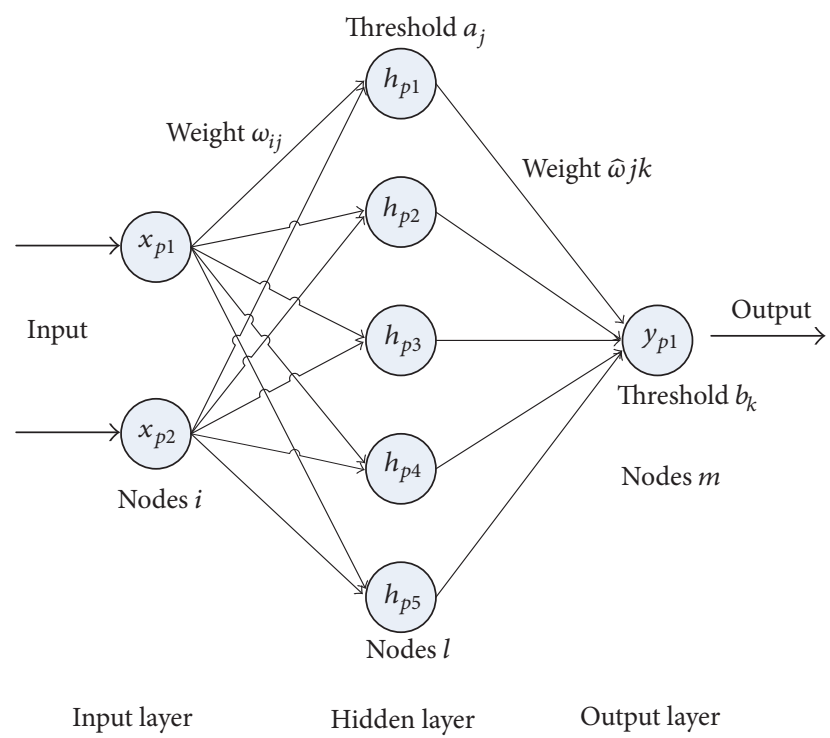

FIgURE 8: The topology of BP neural network.

ability and is the most widely used neural network. BP neural network is mainly through the input data for network training, finally establishes a mapping relationship, and then does network prediction output. The neural network is widely used, for example, in the motion control of an underactuated wheeled inverted pendulum model [25] and robotic selfidentification for surrounding obstacle [26]. The topology of BP neural network is shown in Figure 8. The structure has two input layers nodes, five hidden layers nodes, and one output layer node. Each node of the structure represents a neuron, and the nodes between the layers and the layers are connected by weights. The following is the working principle of BP neural network.

(1) Network Initialization. According to CFD simulation results, determine the network input data and output data $(X$, $Y) . H$ is the data matrix of the hidden layer, as can be seen in

$$
\begin{aligned}
& X_{p}=\left(x_{p 1}, x_{p 2}, \ldots, x_{n p}\right) \\
& H_{p}=\left(h_{p 1}, h_{p 2}, \ldots, h_{p l}\right) \\
& Y_{p}=\left(y_{p 1}, y_{p 2}, \ldots, y_{p m}\right),
\end{aligned}
$$

where $n$ is number of input nodes; $m$ is the number of output nodes; $l$ is number of hidden layer nodes; $p$ is number of training samples. This paper $n$ is equal to $2, m$ is equal to 1 , $l$ is equal to 5 , and $p$ is equal to 32 .

(2) The Output of the Hidden Layer. According to the input vector $X$, the output $h$ of the hidden layer can be calculated, as can be seen in

$$
h_{p i}=f\left(\sum_{i=1}^{n} \omega_{i j} x_{p i}-a_{j}\right), \quad j=1,2, \ldots, l,
$$

where $\omega_{i j}$ is the connection between the input layer and the hidden layer weight and $a_{j}$ is the threshold of the hidden layer nodes. $f(x)$ is the activation function of the hidden layer which is shown in

$$
f(x)=\frac{1}{1+e^{-x}} .
$$

(3) The Output of the Output Layer. Based on output 3 of the hidden layer, the prediction output of BP neural network is calculated, as shown by

$$
\widehat{y}_{p k}=\sum_{j=1}^{l} h_{p i} \widehat{\omega}_{j k}-b_{k}, \quad k=1,2, \ldots, m,
$$

where $\widehat{\omega}_{j k}$ is the connection weight between the hidden layer and the output layer and $b_{k}$ is the threshold of output layer nodes.

(4) Error Calculation. According to the network forecast output $\hat{y}_{k}$ and the expected output $y_{k}$, then calculate the network prediction error $e_{k}$, as shown by

$$
e_{k}=y_{p k}-\widehat{y}_{p k} \text {. }
$$

(5) Weight Update. The weight is updated according to the network prediction error $\left(e_{k}\right)$, as shown in

$$
\begin{aligned}
& \omega_{i j}=\omega_{i j}+\eta h_{p i}\left(1-h_{p i}\right) x(i) \sum_{k=1}^{m} \widehat{\omega}_{j k} e_{k} \\
& \widehat{\omega}_{j k}=\widehat{\omega}_{j k}+\eta h_{p j} e_{k} \\
& \quad \quad \quad \quad i=1,2, \ldots, n ; j=1,2, \ldots, l ; k=1,2, \ldots, m,
\end{aligned}
$$

where $\eta$ is the learning efficiency of BP neural network.

(6) Threshold Update. The threshold is updated according to the network prediction error $\left(e_{k}\right)$, as shown in

$$
\begin{aligned}
& a_{j}=a_{j}+\eta h_{p j}\left(1-h_{p j}\right) \sum_{k=1}^{m} \widehat{\omega}_{j k} e_{k} \\
& b_{k}=b_{k}+\eta e_{k} .
\end{aligned}
$$

Determine whether the iteration is over; if not, return to step (2). It is noted that all the iterative processes of the BP neural network have a mean square error (mse), as shown in (22). In addition, the average accuracy of the prediction $(R)$ is defined by Kreith et al. (2000) [27] and Wang (2000) [28]. In other words, $R$ represents the goodness of fit, which is used to measure the correlation between prediction output data and training samples data. The closer it is to the value of 1 , the better the training network.

$$
\begin{aligned}
\mathrm{mse} & =\frac{1}{m p} \sum_{p=1}^{p} \sum_{j=1}^{m}\left(\hat{y}_{p j}-y_{p j}\right)^{2} \\
R & =\frac{1}{P} \sum_{i=1}^{P} R_{i}=\frac{1}{P} \sum_{i=1}^{P} \frac{y_{p j}}{\widehat{y}_{p j}},
\end{aligned}
$$

where $m$ is the number of output nodes; $p$ is number of training samples. 


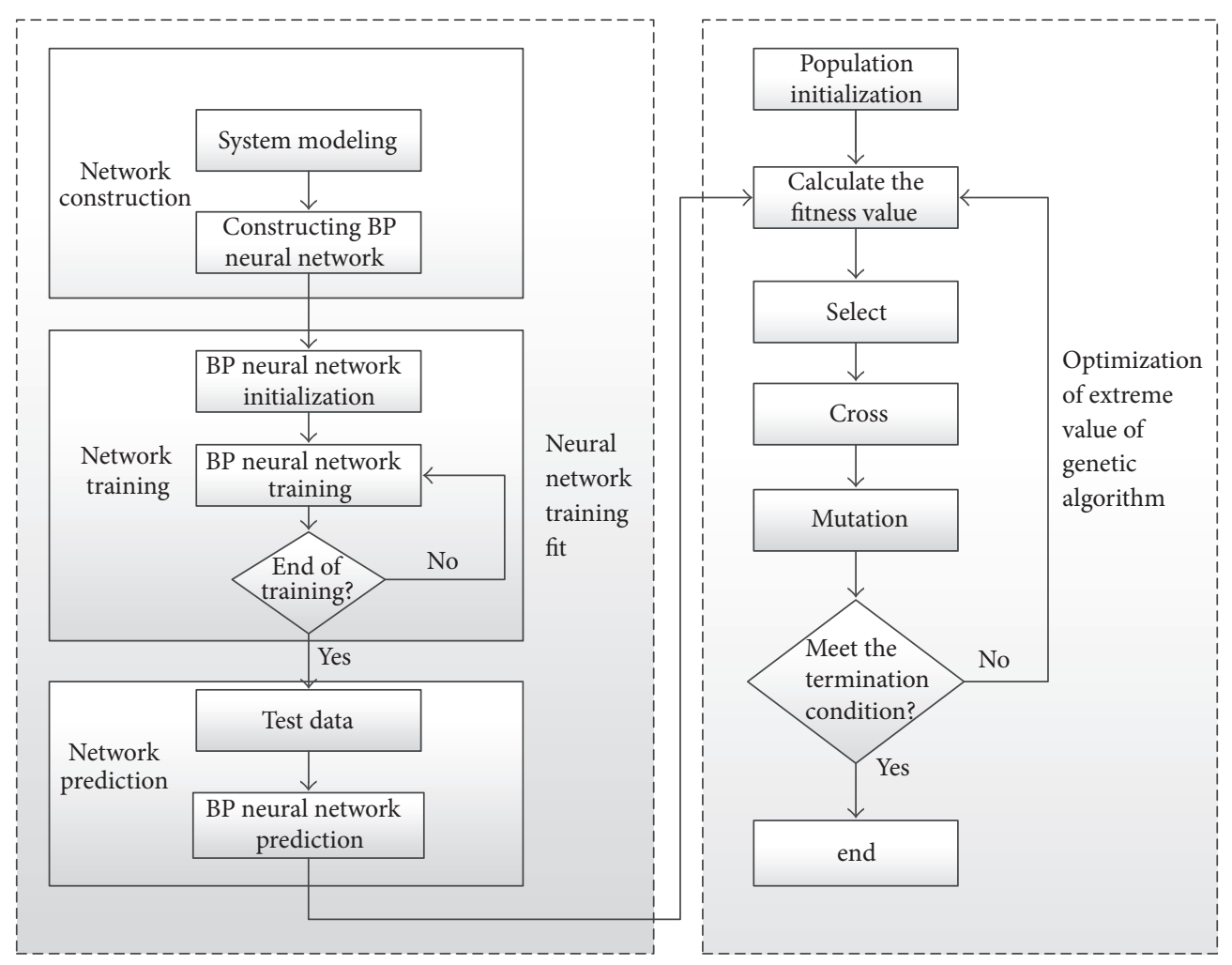

FIGURE 9: Neural network and genetic algorithm flow chart.

5.2. Genetic Algorithm Optimization. Genetic algorithm is a computational model of the biological evolution process of the simulation genetic mechanism. It is a method to search the optimal solution for the feasible solution of the problem. It has strongly global optimization ability. In the genetic algorithm, we will be the individual of each population as a solution to the problem, that is, chromosomes. Genetic algorithm in MATLAB in the operation process is as follows:

(1) First, we initialize the population and then calculate the fitness value. Finally, we look for the best chromosome from the initial population, which is the solution to the problem.

(2) Iterative optimization is as follows:

(a) Select: first, the solution to the problem is encoded by using the floating-point encoding. This function selects the chromosomes in each generation population for subsequent crossover and mutation. The method used is the roulette selection method.

(b) Crossover: this function is a random selection of two chromosomes, according to the crossover probability of determining whether the cross and the cross position are also random.

(c) Mutation: this function performs the mutation operation. The mutation chromosomes and mutated positions are randomly selected. Finally, it will check the feasibility of chromosomes; otherwise, it will be recompiled.

(d) Result analysis.
After several generations, there is an optimal solution to the problem in the population. The establishment of BP neural network's approximate model and the numerical optimization of the genetic algorithm are shown in Figure 9 [29].

\section{Analysis of Vibration Suppression Rate of CFD Simulation Results to the UMP}

6.1. Vibration Amplitude Analysis. As is shown in Table 6, this paper has obtained 33 sets of data by CFD simulation. Through these sets of data, we can analyze the influence of dimensionless plate length $\left(L_{p} / D\right)$ and damping (c) on the amplitude of vibration of UMP, as shown in the three-dimensional Figure 10. The $x$-coordinate of the threedimensional figure represents the change of the dimensionless plate length $\left(L_{p} / D\right)$, the $y$-coordinate represents the change of the damping value $(c)$, and the $z$-coordinate represents the vibration suppression rate $\left(P_{y}\right)$. In addition, it corresponds to the two-dimensional contour line as shown in Figure 11. From these two graphs we can get the effect of the change of dimensionless plate length $\left(L_{p} / D\right)$ and damping value $(c)$ on the vibration suppression rate $\left(P_{y}\right)$. We summarize the following rules.

(1) On the whole, when the damping value is constant, the effect of the dimensionless plate length $\left(L_{p} / D\right)$ on the UMP is increased first and then decreases with the increase of the dimensionless plate length $\left(L_{p} / D\right)$. When the damping values $(c)$ are at $50,75,100,125$, and $175(\mathrm{~N} \times \mathrm{s} / \mathrm{m})$, respectively, the result of the vibration suppression of the 


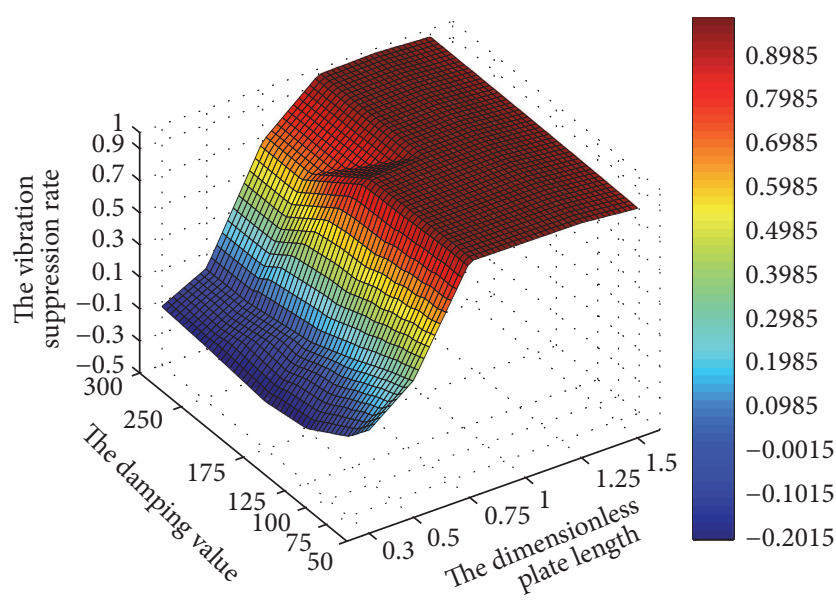

FIGURE 10: Three-dimensional surface cloud diagram of variation of the vibration suppression rate.

UMP is most obvious, reaching more than 0.9 with the change of the dimensionless plate length $\left(L_{p} / D\right)$ from 0.75 to 1.25 .

(2) When the dimensionless plate length $\left(L_{p} / D\right)$ is 0.75 , the suppression rate $\left(P_{y}\right)$ dropped from 0.955698 to 0.671616 , with the damping value $(c)$ changing from 175 to $300(\mathrm{~N} \times \mathrm{s} / \mathrm{m})$. When the dimensionless plate $\left(L_{p} / D\right)$ is 0.3 , the vibration suppression rate $\left(P_{y}\right)$ drops to a negative value, which indicating that it will not suppress the vibration of the $\mathrm{UMP}$ in this case. In the dimensionless plate length $\left(L_{p} / D\right)$ at $0.3,0.5,0.75$, and 1.0 , respectively, the vibration suppression rate $\left(P_{y}\right)$ decreases with the increase of the damping value, but it is increasing when the dimensionless plate length $\left(L_{p} / D\right)$ is 1.5 . When the dimensionless plate length $\left(L_{p} / D\right)$ is 1.25 , the vibration suppression rate $\left(P_{y}\right)$ increases first and then decrease with the increase of the damping value, and the maximum vibration suppression rate $\left(P_{y}\right)$ is 0.979676 .

(3) In these data, when $L_{p} / D=1.25, c=175(\mathrm{~N} \times \mathrm{s} / \mathrm{m})$ is the best data point of the UMP vibration suppression. Its vibration suppression rate $\left(P_{y}\right)$ reached 0.979676 .

6.2. Analysis of Lift Coefficient and Drag Coefficient. We study the two different parameters separately and discuss the variation law of the lift coefficient and the drag coefficient of a parameter to the whole UMP, respectively. It should be noted here that the lift coefficient we are studying is the total lift coefficient of whole system. As the drag coefficient of the plate relative to the cylinder drag coefficient is very small, we ignore the plate drag coefficient and the cylinder drag coefficient approximation as the whole system drag coefficient. In order to clearly characterize the force of the whole system, we chose the root mean square (RMS) of the lift coefficient and the averaged drag coefficient in the following study.

(1) Influence of Dimensionless Plate Length on Lift Coefficient and Drag Coefficient. Figure 12 shows the variation of the total lift coefficient between different damping values as the dimensionless plate length increases. At the same time, we compare it with the lift coefficient of the bare cylinder.
As can be seen from figure, overall, in the case of the same damping value, the RMS of total lift coefficient decreases first and then increases gradually with the increase of the dimensionless plate length. It can be seen that when the dimensionless plate length is from 0.5 to 0.75 , the RMS of total lift coefficient sharply is down from about 0.6 to about 0.15 . When the dimensionless plate length is $0.75,1,1.25$, and 1.5, their RMS of total lift coefficient are less than the bare cylinder. And we can see that the damping value is $175(\mathrm{~N} \times \mathrm{s} / \mathrm{m})$ and the dimensionless plate length is 1.25 when the RMS of total lift coefficient is the smallest.

As can be seen from Figure 13, all the mean drag coefficient of the cylinder is less than the bare cylinder. The drag coefficient is reduced with the increase of dimensionless plate length. When the dimensionless plate length is $0.75,1.0,1.25$, and 1.5, the larger the damping value, the smaller the mean drag coefficient, but the opposite when the dimensionless plate length is 0.3 and 0.5 .

(2) Influence of the Damping Value on Lift Coefficient and Drag Coefficient. Figure 14 is a graph showing a corresponding changes in the RMS of total lift coefficient when the damping value is increased. It is clear from Figure 15 that the RMS of total lift coefficient is much larger than that of the bare cylinder when the dimensionless plate length is 0.3 and 0.5 over the entire damping value change region. The remaining dimensionless plate length is less than the bare cylinder or close to it. In the range where the damping value is greater than 100, the RMS of total lift coefficient of the dimensionless plate length equal to 1.25 is smaller than that of the other dimensionless plates, and when the damping value is $175(\mathrm{~N} \times \mathrm{s} / \mathrm{m})$, the RMS of total lift coefficient is minimized and the minimum value is 0.048405 . The dimensionless plate length 0.75 is relatively variable, and when the damping value is 50 and 75 , the RMS of total lift coefficient is the smallest compared to the other dimensionless plate length. When the damping value changes from 175 to $250(\mathrm{~N} \times \mathrm{s} / \mathrm{m})$, the RMS of total lift coefficient increases dramatically.

In Figure 15, we can see that the mean drag coefficient of the bare cylinder is the largest compared with the all dimensionless plate length when the damping value increases. The mean drag coefficient of the dimensionless plate length of 0.3 and 0.5 increased gradually as the damping value increases. However, the remaining dimensionless plate length is reduced. The longer the dimensionless plate length is, the smaller the mean drag coefficient in the region of the global damping range will be.

Through the above analysis, we can get the following conclusions.

(1) When the RMS of total lift coefficient of the entire system is less than the RMS of lift coefficient of the bare cylinder, the vibration suppression effect is obvious, and the vibration suppression rate is more than 0.9 .

(2) The mean drag coefficient of the cylinder with the plate is smaller than the bare cylinder. That is to say, the plate can reduce the drag force.

6.3. Analysis of Wake Structure. Through the numerical simulation results, when the dimensionless plate length is 1.25 


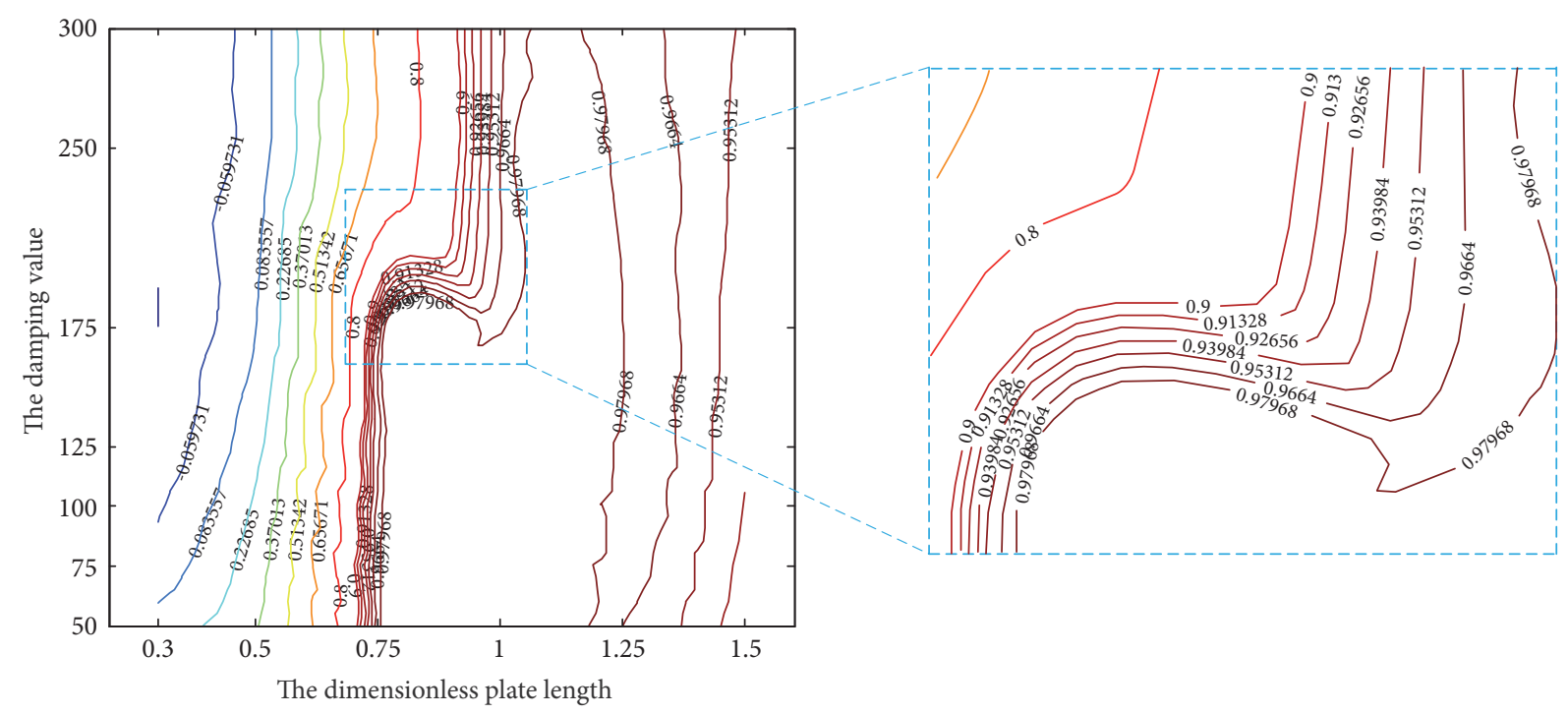

FIGURE 11: Two-dimensional contour line of variation of the vibration suppression rate.

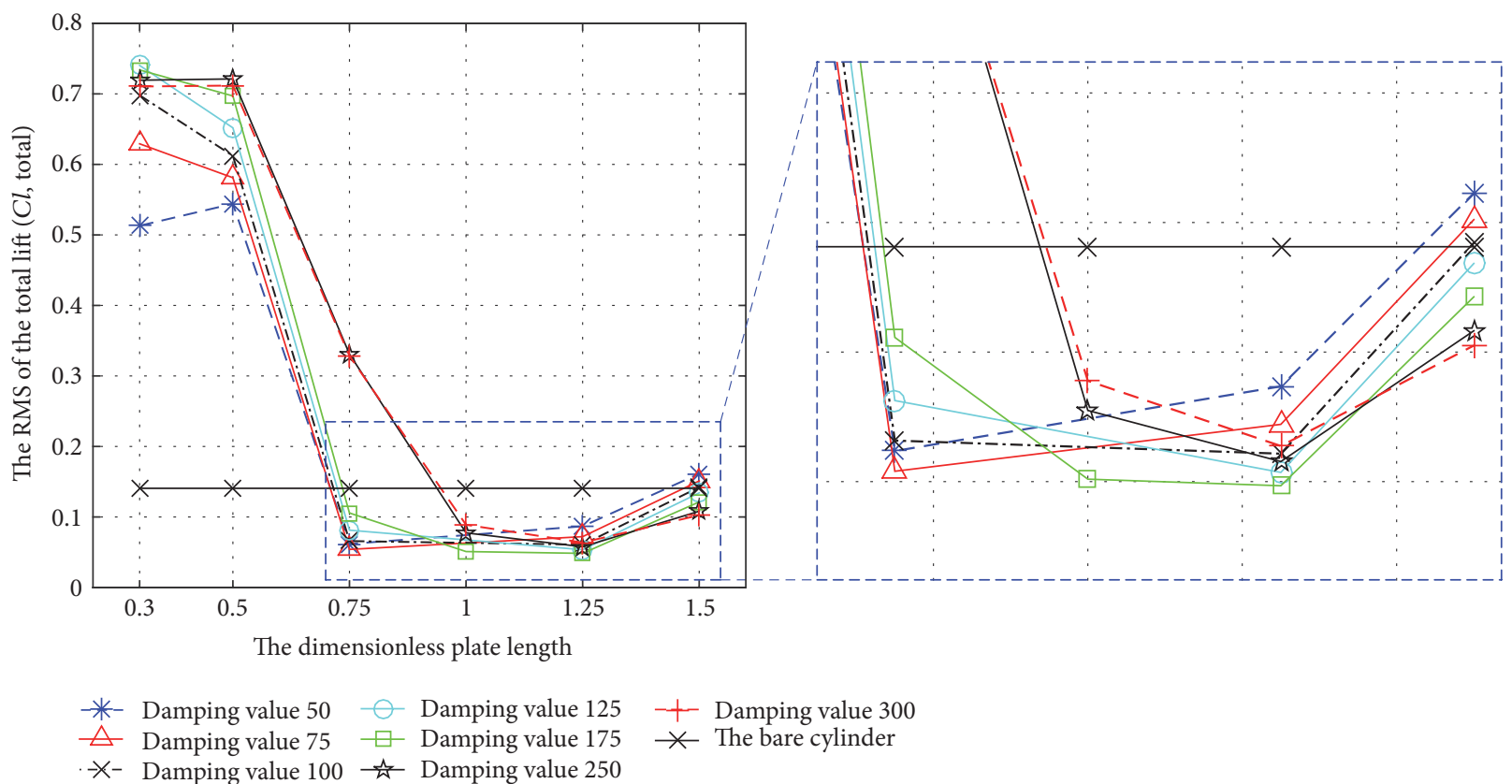

FIGURE 12: The variation of the total lift coefficient (RMS) with the changing of the dimensionless plate length.

and the damping value is 175 , the plate has the best effect of suppressing the vibration of the UMP. So we chose the case to analyze its vortex pattern and vortex shedding. Vortex shedding patterns can refer to the literature of Williamson and Roshko (1988) [30], and there are 2S, 2P, and 2T.

As can be seen from Figure 16, the graph is a contours of vorticity with a dimensionless plate length of 1.25 and a damping value of $175(\mathrm{~N} \times \mathrm{s} / \mathrm{m})$. The contour of vorticity shows that its structure is a typical $2 \mathrm{~S}$ vortex pattern. This shows that the plate for the UMP wake region has a certain impact. The shear layer is then extended to the tip of the plate.
Figure 17 is a description of the interval of $(1 / 4) T$ cycles and then draws the different interval cycle contours of vorticity and pressure. As can be seen from the figure, the plate prevents the interaction between the shear layers. The vortex shedding extends from the surface of the cylinder to the wake end of the plate. So that the pulsating force on the cylindrical surface is weakened. As can be seen from the vorticity cloud, the vortex is moving from the root of the plate to the tip. On the sides of the plate there is always only one vortex, one vortex is at the same time as the tip of the plate, and the other vortex is formed at the root of the plate. From 


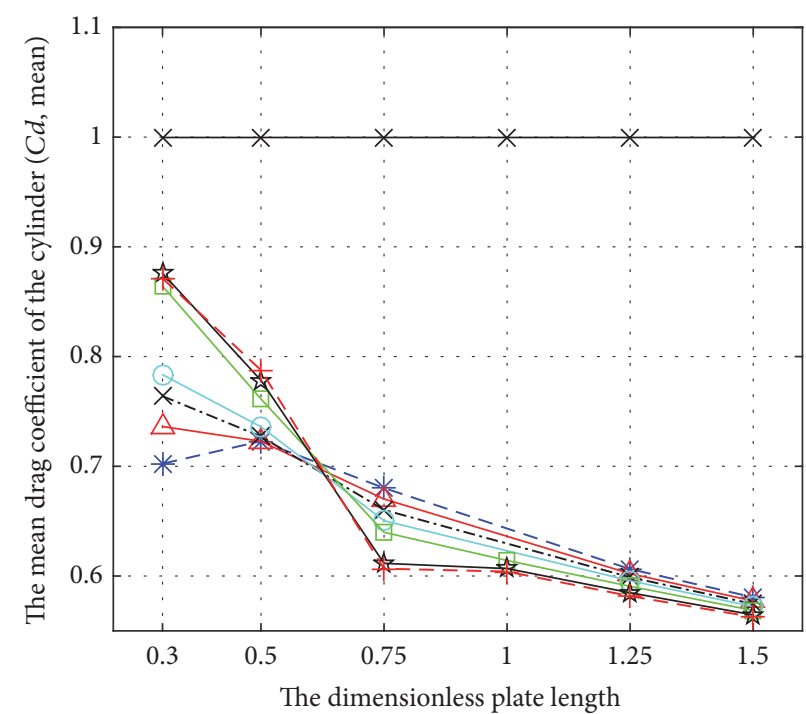

$$
\begin{aligned}
& \text { - * Damping value 50 - Damping value } 125 \text { - } \bigcirc \text { Damping value } 300 \\
& \triangle \text { Damping value } 75 \quad \square \text { Damping value } 175 \quad \chi \text { The bare cylinder } \\
& -X \text { - Damping value } 100 \quad \frac{1}{\sqrt{2}} \text { Damping value } 250
\end{aligned}
$$

FIgURE 13: The variation of the drag coefficient (mean).

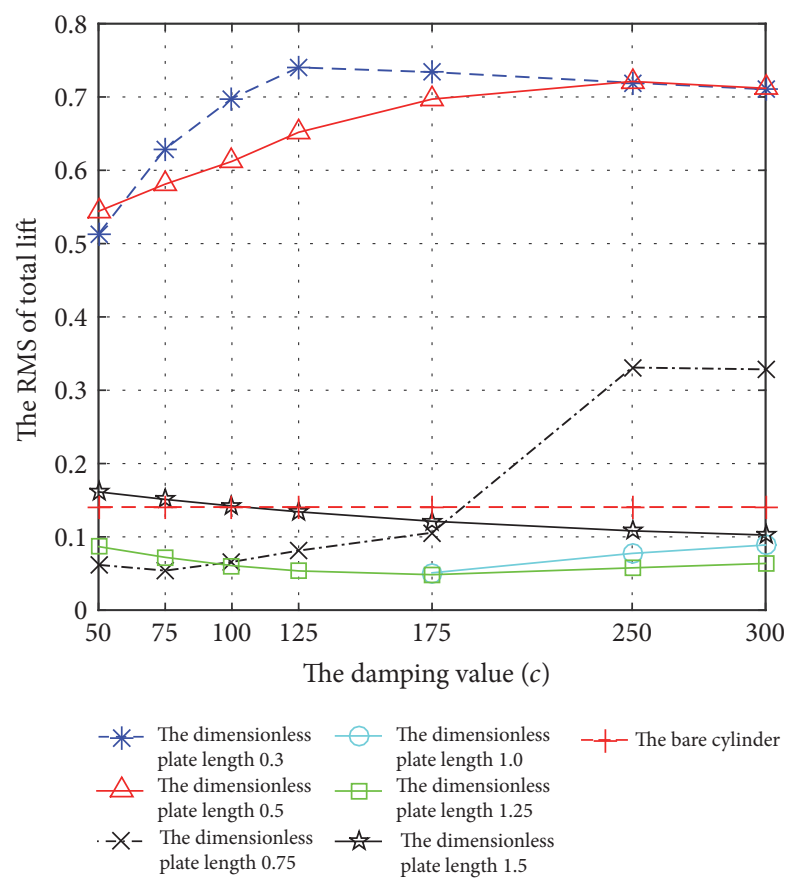

FIGURE 14: The variation of the RMS of total lift coefficient with the changing of the damping value.

the pressure cloud shows that the vortex of the cylindrical surface can not fall off the rear surface of the cylinder for the presence of the partition plate but is extended to the tip of the partition plate. At this time from the figure can be seen, the cylindrical surface of the back pressure on both sides is difficult to change. It was changed significantly at the end of the board, and the pressure on the surface of the cylinder was

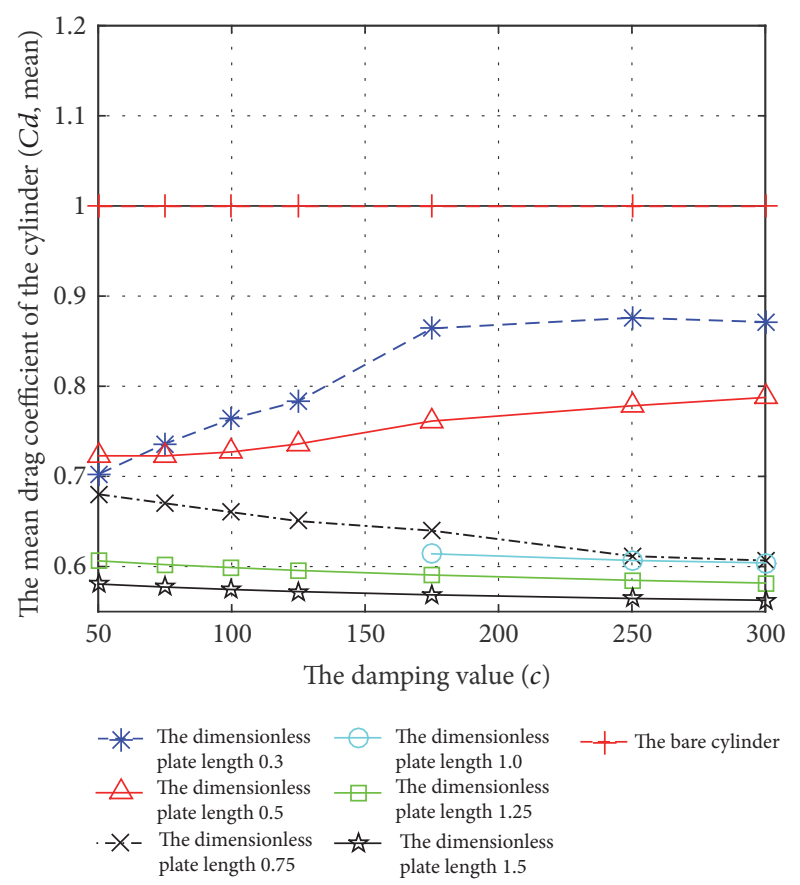

FIGURE 15: The variation of the mean drag coefficient with the changing of the damping value.

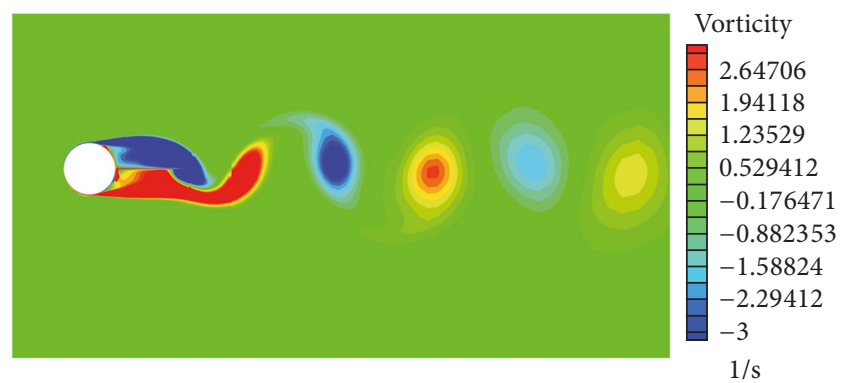

FIGURE 16: A contour of vorticity with a dimensionless plate length of 1.25 and a damping value of 175 .

observed to be opposite to the pressure of the plate. This helps to reduce lift.

\section{Neural Network and Genetic Algorithm Optimization}

\subsection{Neural Network Training}

(1) Selection of Sample Data. In this study, we use MATLAB software as a neural network and genetic algorithm optimization platform. First, we have to select the reasonable sample data points. As can be seen from Table 6, the suppression rate of the third to eight data is negative. This situation does not achieve the effect of vibration suppression; it can not be used as the optimization of data points. The first group is bare cylindrical data, so it can not be used as an optimized 

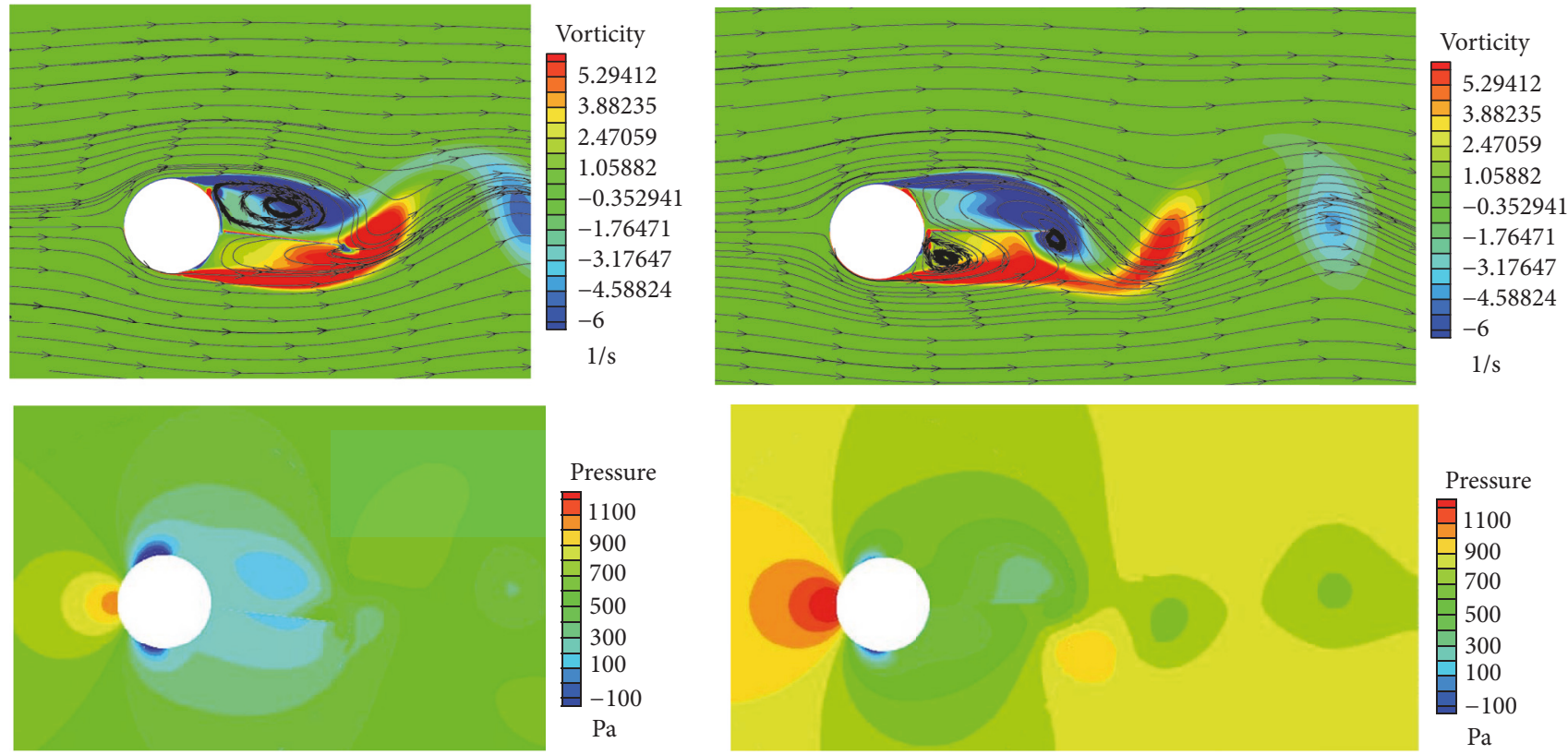

(a) $t=(1 / 4) T$

(b) $t=(1 / 2) T$
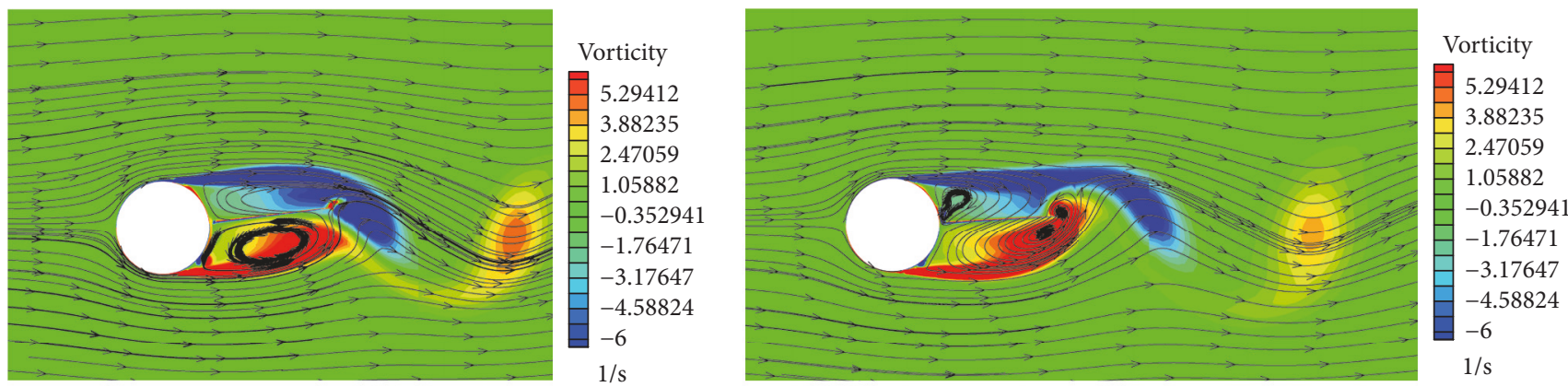

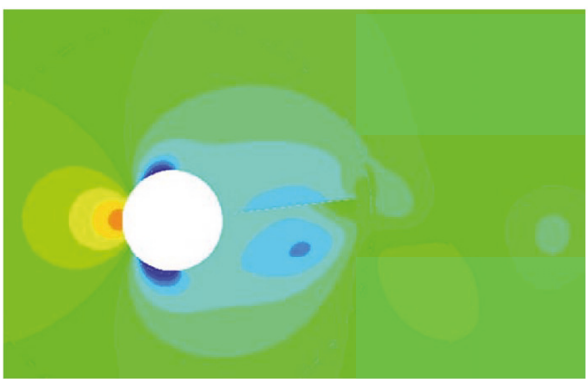

(c) $t=(3 / 4) T$

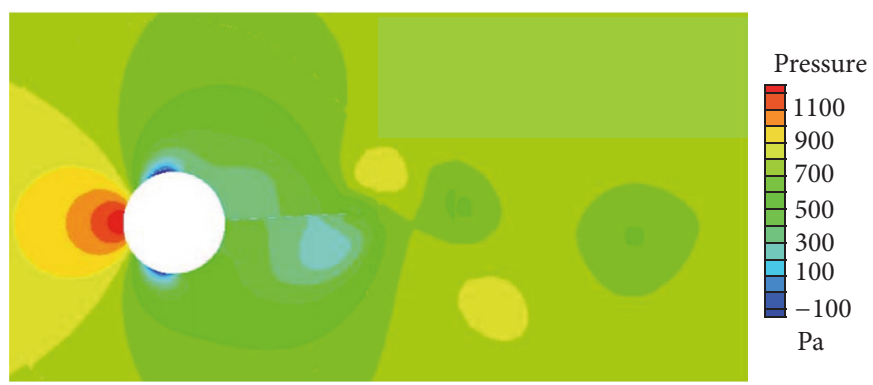

(d) $t=T$

FIGURE 17: The different interval cycle contours of vorticity and pressure.

sample point. Among them, we use random 19 sets of data for training fata, the remaining 13 sets of data as test data.

(2) Network Model Establishment. From Figure 7 we can see that we use 3-layer neural network to process 32 sets of data, and the number of hidden layer take 5 .

(3) Network Training. We set the target error of the network (net.trainParam.goal) to 0.000004 , the learning rate
(net.trainParam.lr) to 0.1 , and the number of training steps (net.trainParam.epochs) to 100. At the same time, we set the mean squared errors (mse) as a performance function.

According to the above settings, the training of BP neural network is carried out until the training network meets the intended target. As a result, the prediction of the BP neural network is shown in Figure 18. As can be seen from the figure, the training network is very close to the date. Table 7 shows the comparison of the neural network prediction output with 
TABLE 7: Comparison of the neural network prediction output with the test sample data points.

\begin{tabular}{lcccc}
\hline Number & Test sample data & Prediction data & Absolute error & Relative error (\%) \\
\hline$(1)$ & 0.949309 & 0.9415 & 0.007809093 & 0.822608 \\
$(2)$ & 0.009622 & 0.01069 & 0.001067986 & 11.099397 \\
$(3)$ & 0.978471 & 0.9766 & 0.001870826 & 0.191199 \\
$(4)$ & 0.971677 & 0.8949 & 0.076777254 & 7.901518 \\
$(5)$ & 0.358304 & 0.3872 & 0.028896097 & 8.064689 \\
$(6)$ & 0.249475 & 0.2568 & 0.007325429 & 2.936343 \\
$(7)$ & 0.955698 & 0.7909 & 0.164798081 & 17.243739 \\
$(8)$ & 0.977124 & 0.931100 & 0.046023748 & 4.710125 \\
$(9)$ & 0.038191 & 0.065120 & 0.026928866 & 70.510779 \\
$(10)$ & 0.965197 & 0.10399661 & 10.774656 \\
$(11)$ & 0.006293 & 0.008060 & 0.014353191 & 228.074937 \\
$(12)$ & 0.673212 & 0.579600 & 0.093611759 & 13.905247 \\
$(13)$ & 0.966174 & 0.970000 & 0.003826088 & 0.396004 \\
\hline
\end{tabular}

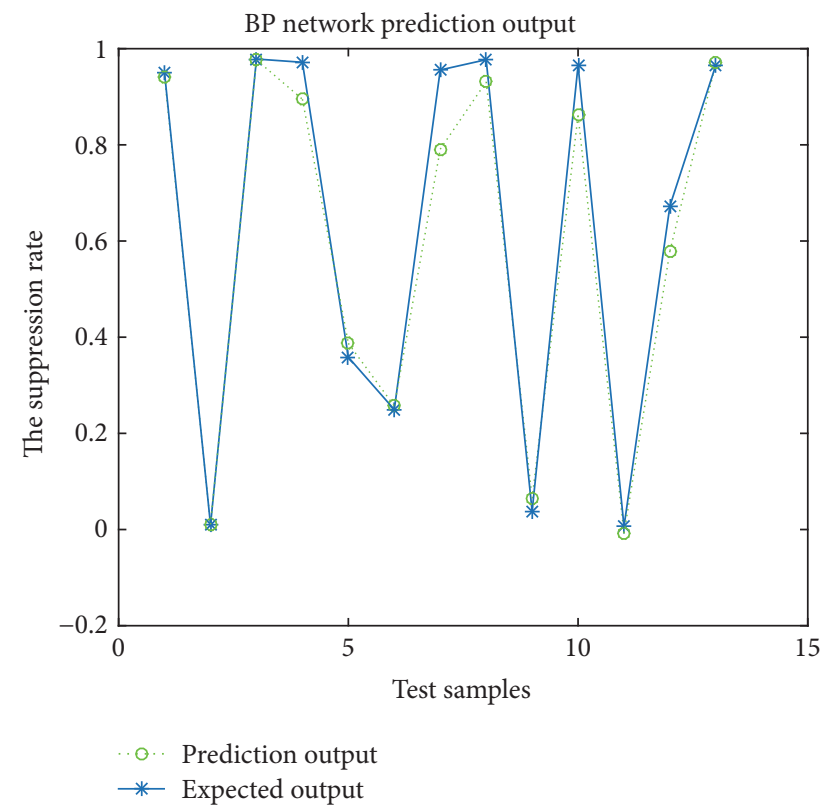

FIGURE 18: BP neural network prediction results. the test sample data points. The maximum absolute error between them is 0.164798081 and the minimum relative error is 0.191199 percent. We can get the relative error in the range of 0.191199 to 228.074937 percent. What needs to be explained here is that we can see that the predicted output data is negative, indicating that the condition does not play the role of vibration suppression but contributed to the vibration. So it is possible that a negative suppression vibration rate with the lower vibration suppression rate in the sample data appears. So its relative error is very large, reaching 228.074937 percent.

Figure 19 shows the performance of the neural network. This figure shows that the Validation Performance is $5.0799 e-$ 5 at epoch 8 , and it has been very close to our target error of the network. Figure 20 shows the regression analysis of the BP

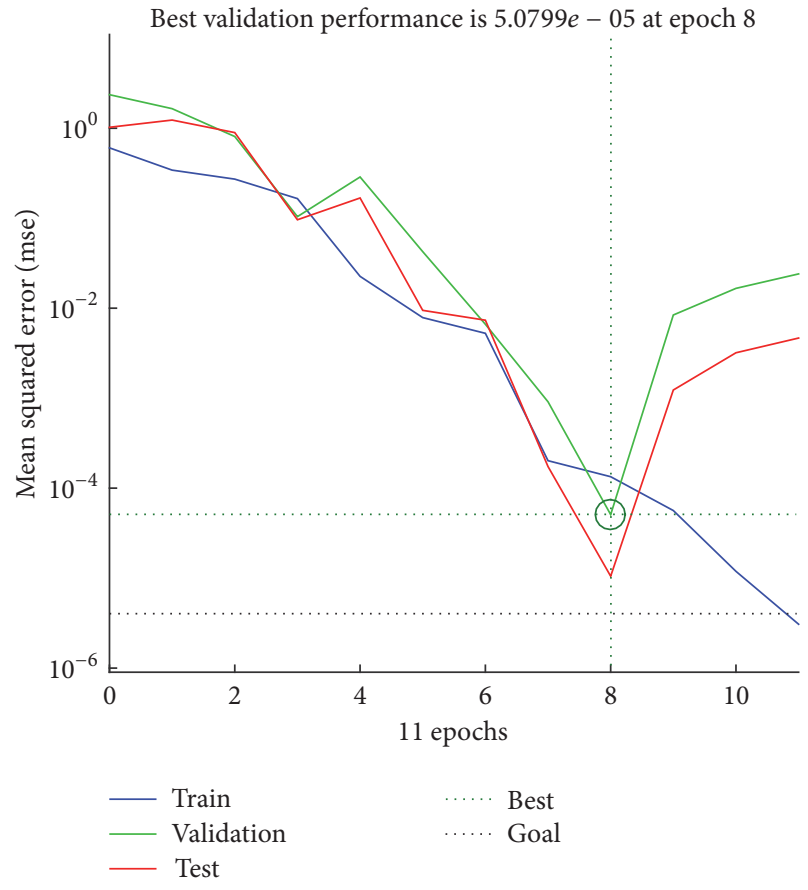

FIGURE 19: The performance of the neural network.

neural network. As can be seen from the figure, the training set, the validation set, and the test set of complex correlation number $(R)$ are very close to 1 . It is shown that the constructed $\mathrm{BP}$ neural network has high prediction accuracy.

7.2. Genetic Algorithm Optimization. Then we use the genetic algorithm to optimize the previously trained BP neural network. The suppression rate of the UMP is taken as the fitness. The fitness curve calculated by the genetic algorithm is shown in Figure 21

As can be seen from Figure 21, when the iteration to the beginning is very fast convergence, the optimal fitness value $\left(P_{y}\right)$ tends to be stable, and its value is 0.9878 . At this 

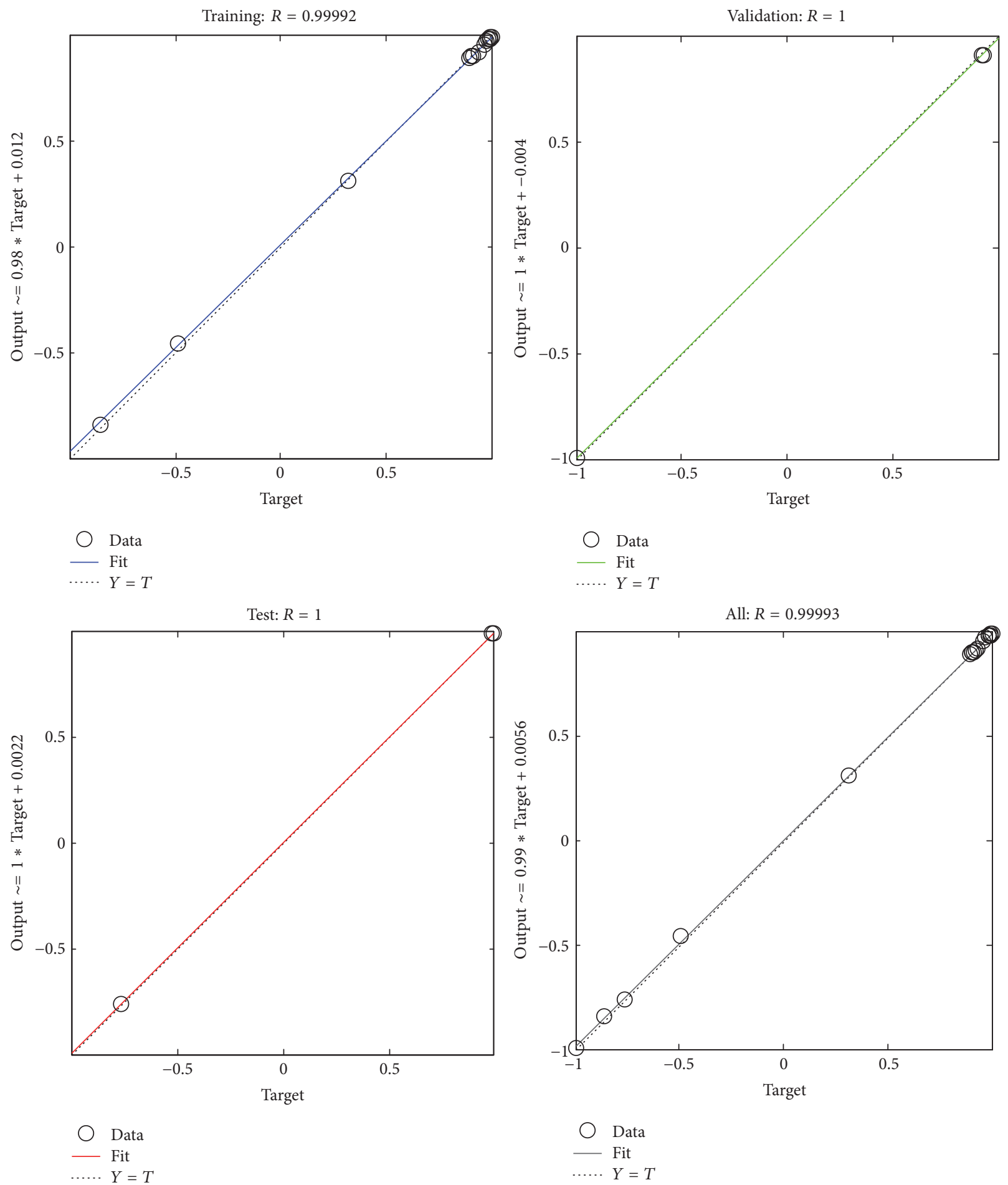

FIGURE 20: The regression analysis of the BP neural network.

time we can get an optimized optimal vibration suppression rate $\left(P_{y}\right)$ as well as the corresponding dimensionless plate $\left(L_{p} / D\right)$ length and damping value $(c)$. They are $P_{y}=0.9878$, $L_{p} / D=1.0342$, and $c=57.9631(\mathrm{~N} \times \mathrm{s} / \mathrm{m})$, respectively. The comparison data are shown in the Table 8. Optimization before and after the data comparison shows that the suppression rate increased by 0.82925 .

\section{CFD Validation}

In order to verify the accuracy and validity of BP neural network and genetic algorithm optimization results, we have obtained the optimization results and verified the optimized results with FLUENT 15.0 numerical test method. At the same time, we must guarantee the same simulation conditions as 


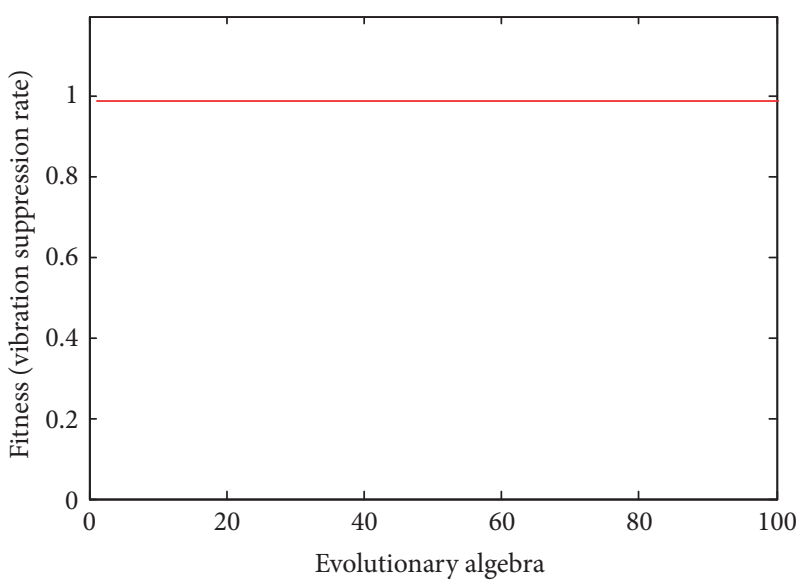

FIGURE 21: The fitness curve calculated by the genetic algorithm.

TABLE 8: Comparison of data before and after optimization.

\begin{tabular}{lcc}
\hline Parameter & Before optimization & After optimization \\
\hline$L_{p} / D$ & 1.25 & 1.0342 \\
$c(\mathrm{~N} \times \mathrm{s} / \mathrm{m})$ & 175 & 57.9631 \\
$P_{y}$ & 0.979676 & 0.9878 \\
\hline
\end{tabular}

TABLE 9: Comparison of the results of the optimized data and CFD simulation.

\begin{tabular}{cccc}
\hline & Optimization results & CFD simulation results & Absolut error \\
\hline$P_{y}$ & 0.9878 & 0.9841 & 0.0037 \\
\hline
\end{tabular}

this article to carry out CFD simulation. The final simulation results are shown in Table 9.

As can be seen from Table 9, the results of BP neural network and generic algorithm optimization are closed to those of CFD simulation, and the absolute error is only 0.0037 . It is shown that the BP neural network and generic algorithm are effective. We also found that the results of the vibration suppression rate optimized by the BP neural network and generic algorithm $\left(P_{y}=0.9878\right)$ are larger than $P_{y}=$ 0.979676 of the samples we use, reaching the highest suppression vibration rate. Similarly, the vibration suppression rate is $P_{y}=0.9841$ under the CFD verification with the optimal parameters $\left(L_{p} / D=1.0342, c=57.9631(\mathrm{~N} \times \mathrm{s} / \mathrm{m})\right)$, which is also the maximum of the sample data. This shows that the BP neural network and generic algorithm can be based on our objective function (vibration suppression rate) to optimize the parameters to achieve the optimal value.

8.1. Comparing the Result of Bare UMP and CFD Simulation Based on Optimal Parameters. We compare the results of the analysis of bare UMP and CFD simulation based on optimal parameters, mainly for dimensionless amplitude, wake structure, and total lift coefficient.

(1) Dimensionless Amplitude. As shown in Figure 22, it is clear that the dimensionless amplitude of the UMP with plate $\left(L_{p} / D=1.0342\right)$ is much smaller than that of the bare UMP.

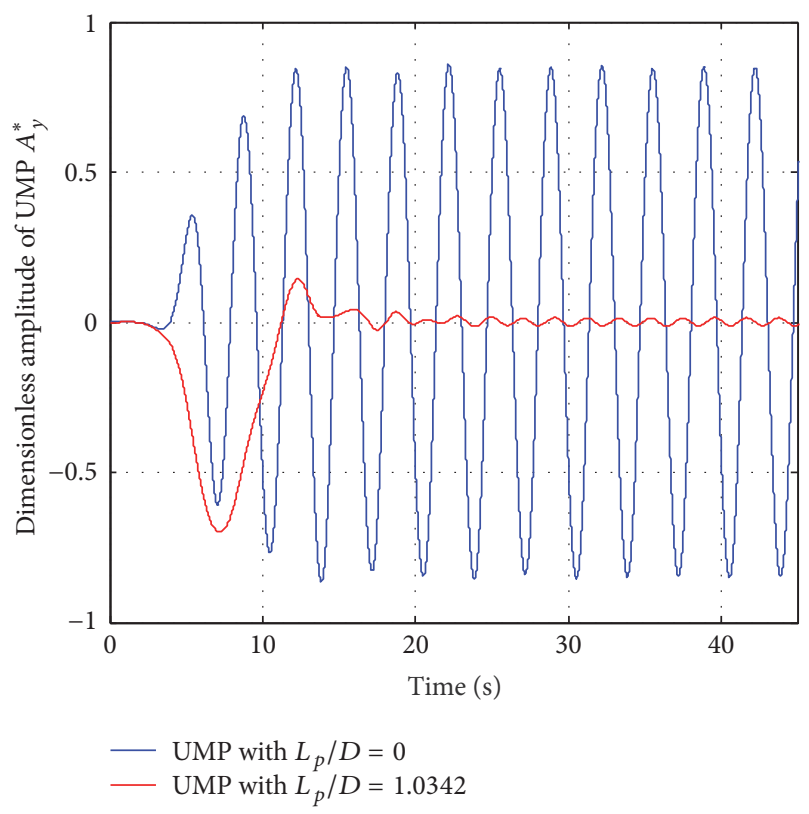

FIGURE 22: Dimensionless amplitude of the bare UMP and UMP with plate based on optimal parameter.

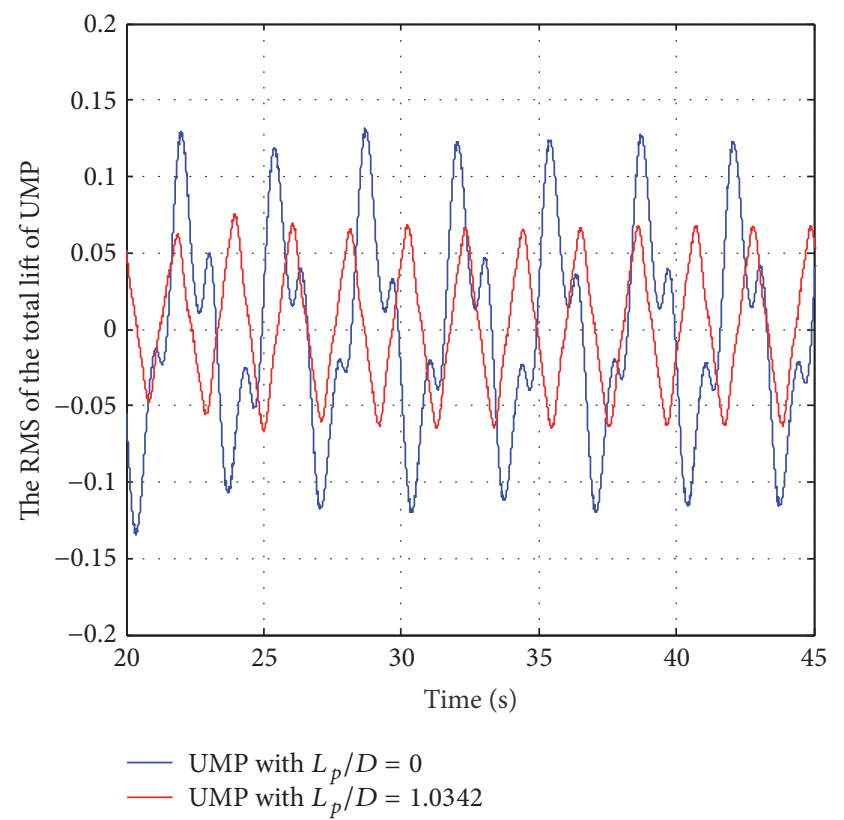

FIgURE 23: The RMS of total lift coefficient of the UMP with plate and the bare UMP.

The dimensionless amplitude s of the UMP with plate and the bare UMP are 0.013508 and 0.847339 , respectively. The suppression rate of the CFD simulation based on optimal parameters is 0.9841 .

(2) The RMS of the Total Lift. As shown in Figure 23, the RMS of total lift coefficient is also reduced relative to the bare UMP by comparing the CFD simulation results with the optimization results (obtained from the BP neural network 

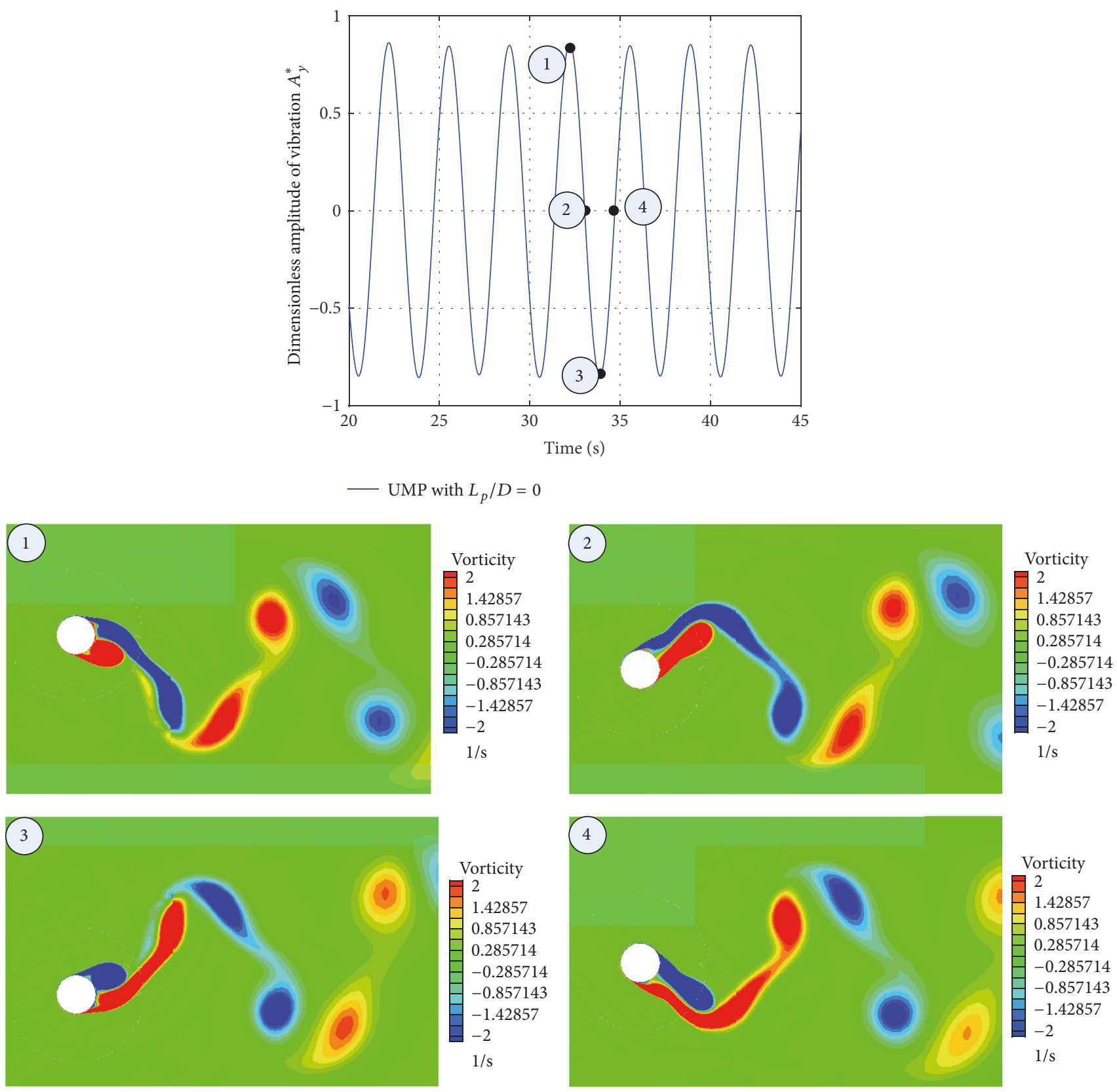

FIGURE 24: The vorticity cloud images of bare UMP.

and genetic algorithm). The RMS of total lift coefficient of the UMP with plate and the bare UMP are 0.040929 and 0.140653 , respectively.

(3) Wake Structure. As shown in Figures 24 and 25, they are the vorticity cloud images of bare UMP and UPM with $L_{p} / D=1.0342$, respectively. We only selected the four points to show vorticity cloud images. It was observed that the plate changed the wake structure pattern of the UMP, and the wake structure pattern changed from the $2 \mathrm{P}$ mode of the bare UMP to the $2 \mathrm{~S}$ mode of the UMP with the plate. The interaction of the shear layer is prevented by the plate. The plate extends the vortex shedding, as the vortex is removed from the UMP and reattached to the plate.

Figures 26(a) and 26(b) are a pressure cloud with the same position as the above vortex cloud. The overall can be seen in Figure 26(a), and the bare UMP's wake region pressure changes significantly. At this point, the front portion of the bare UMP by the pressure distribution is not uniform. So it produced a relatively large lift. On the contrary, in Figure 26(b), the pressure on both sides of the plate behind the UMP has not changed greatly, because the vortex is extended to the tip of the plate to shed in the wake region of the UMP with the plate. 


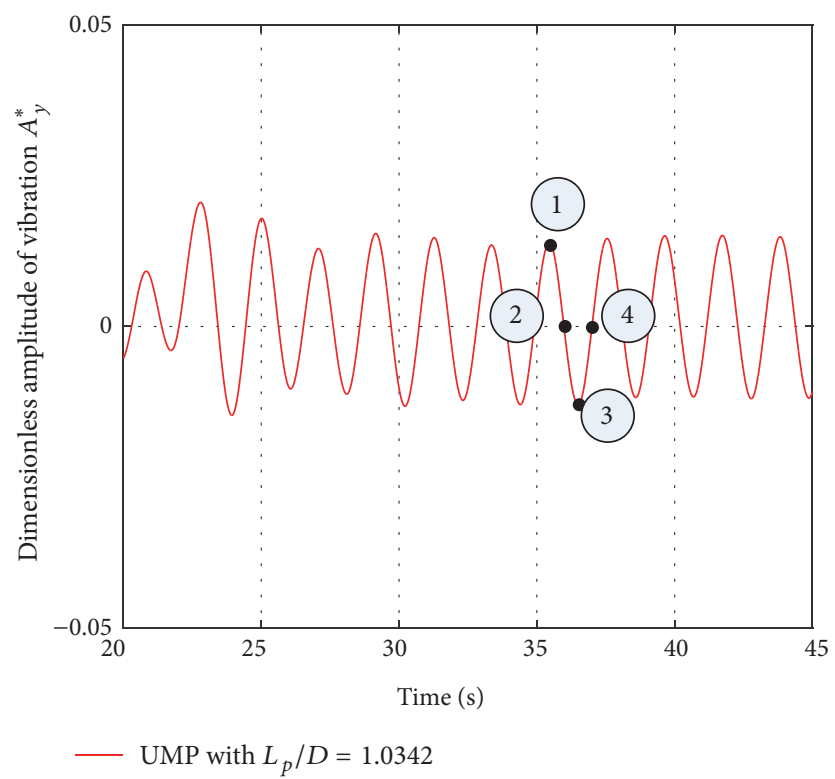

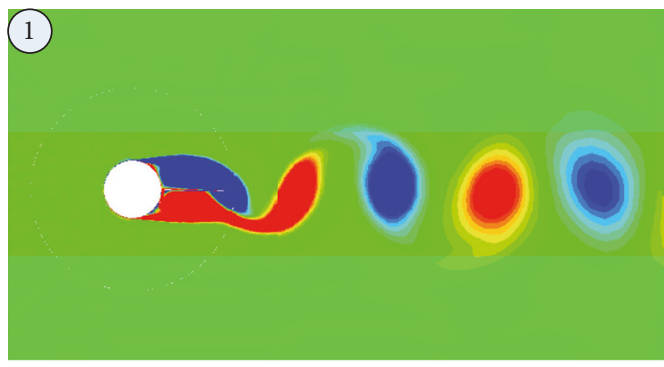
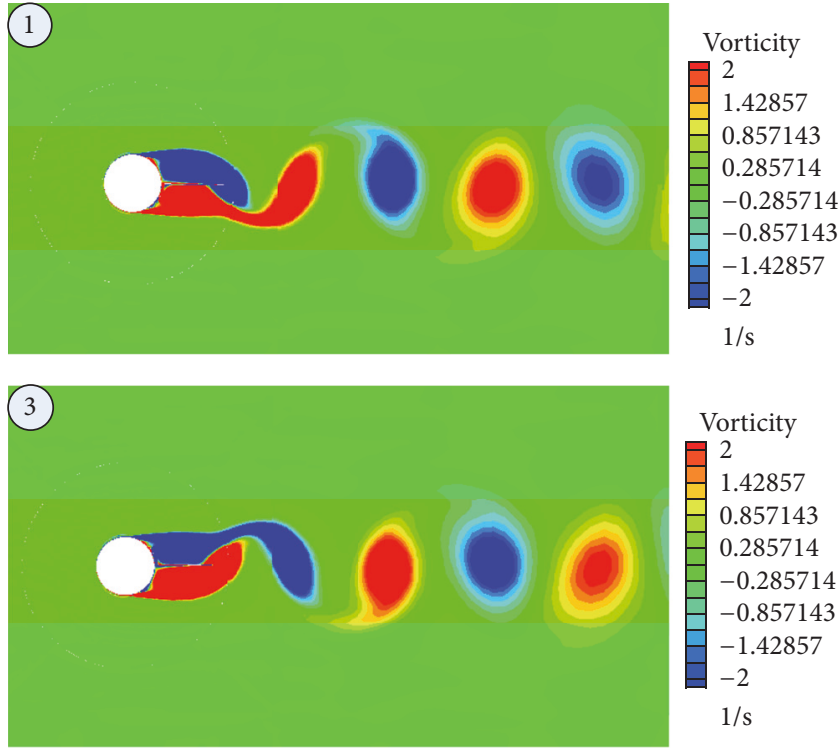
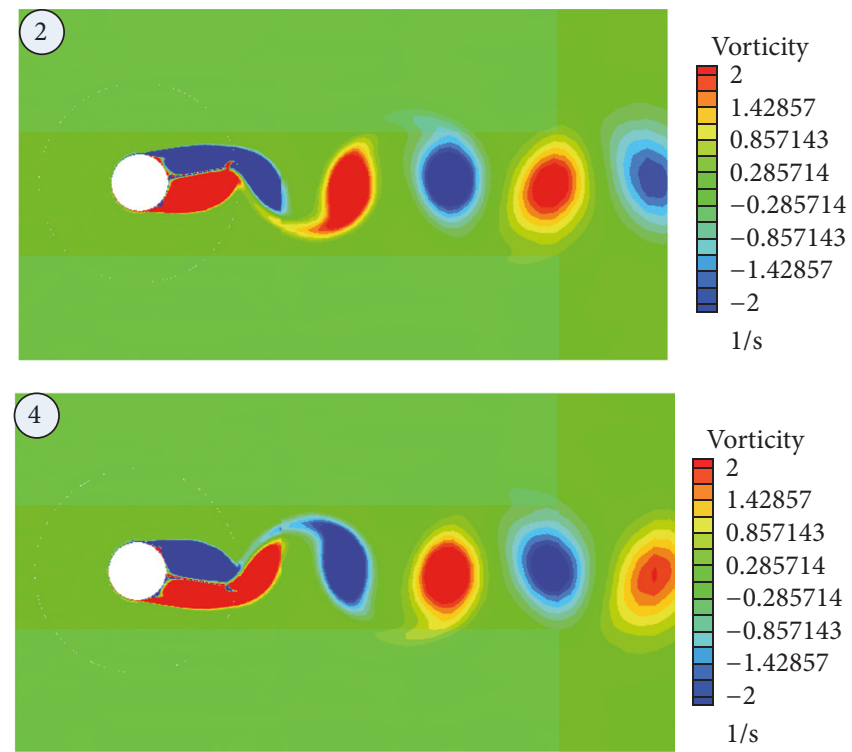

FIGURE 25: The vorticity cloud images of UPM with $L_{p} / D=1.0342$.

\section{Conclusion}

Under the action of current flow, the UMP suspended in seawater will undergo vortex-induced vibration, so that it can lose its value. In this paper, we studied the effect of splitter plate on the vibration of the UMP. The main parameters of the plate are the dimensionless plate length $\left(L_{p} / D\right)$ and the damping value $(c)$ between the UMP and plate. For us to use CFD numerical simulation of the UMP vibration, it will increase the calculation cost and time with the more situations. So, we consider using BP neural network and generic algorithm to optimize these two parameters in order to minimize the vibration of the UMP. For this study, the following conclusions can be drawn.

(1) We selected 38 sample data points for CFD simulation and extracted the results. From the finite discrete parameter data point, we can see that the amplitude of the UMP is the smallest when the dimensionless plate length $\left(L_{p} / D\right)$ is 1.25 , the damping $(c)$ is $175(\mathrm{~N} \times \mathrm{s} / \mathrm{m})$, and the maximum suppression rate $\left(P_{y}\right)$ is 0.979676 . The effect on the suppression vibration achieves the best results. When the dimensionless plate length is 0.3 and 0.5 , regardless of the fact that the damping value is how much, its vibration suppression effect is poor and may increase the vibration amplitude. The vibration suppression effect is better in the dimensionless plate length of 0.75 to 1.5 , and the best damping ranges vary with dimensionless plate length.

(2) We use MATLAB software as a neural network and genetic algorithm optimization platform. First, we selected the appropriate 32 sets of data as an optimized variable and the suppression rate as the objective function for BP neural network and genetic algorithm. The optimization results 

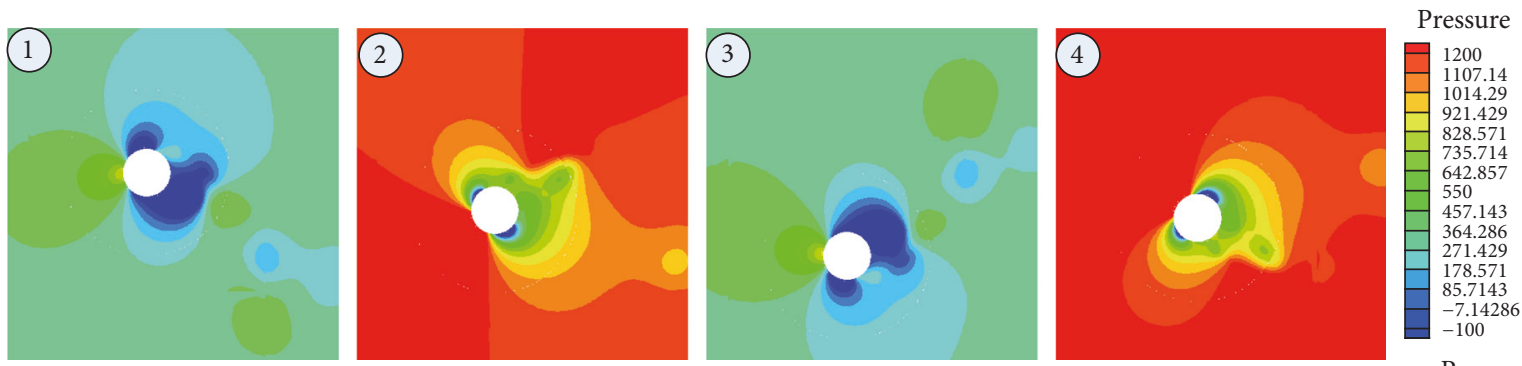

(a) The pressure contour of UMP with $L_{p} / D=0$
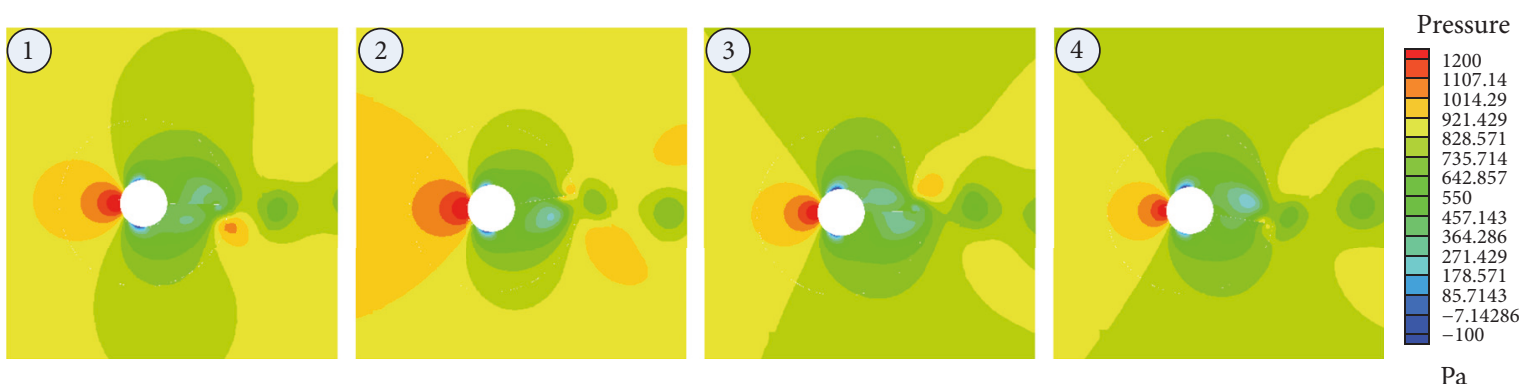

(b) The pressure contour of UMP with $L_{p} / D=1.0342$

FIGURE 26: Pressure cloud of the bare UMP and UMP with plate.

show that the optimum suppression rate is 0.9878 , and the corresponding dimensionless plate length $\left(L_{p} / D\right)$ is 1.0342 and the damping value $(c)$ is $57.9631(\mathrm{~N} \times \mathrm{s} / \mathrm{m})$.

(3) In order to verify the accuracy of the optimization, we perform the CFD numerical simulation with the optimized parameters and compare the theoretical optimization results with the CFD simulation result. As can be seen from Table 9, the results of BP neural network and generic algorithm optimization are closed to those of CFD simulation, and the absolute error is only 0.0037. It is shown that the BP neural network and generic algorithm are effective.

Through the study of this paper, we obtained the parameter value when the vibration suppression effect is the best by BP neural network and genetic algorithm and CFD validation.

\section{Conflicts of Interest}

The authors declare that they have no conflicts of interest.

\section{Acknowledgments}

This research was supported by the National Science Foundation of China (Grants nos. 51179159 and 61572404) and the Shaanxi Province Youth Science and Technology New Star Project (Grant no. 2016KJXX-57).

\section{References}

[1] L. Ding, L. Zhang, Z.-Q. Yang et al., "Effect of splitter plate on vortex-induced vibration of circular cylinder at high Reynolds number," Chinese Journal of Mechanical Engineering, vol. 49, no. 14, pp. 133-139, 2013.
[2] B. Tan, J.-S. Wang, F. Gu et al., "Numerical simulation of vortexinduced vibration of riser using separation disk," Hydrochemistry Research and Progress, vol. 24, no. 1, pp. 43-48, 2009.

[3] J. Wang, H. Liu, F. Gu, and P. Zhao, "Numerical simulation of flow control on marine riser with attached splitter plate," in Proceedings of the ASME 2010 29th International Conference on Ocean, Offshore and Arctic Engineering, pp. 489-498, June 2010.

[4] M. Amiraslanpour, J. Ghazanfarian, and S. E. Razavi, "Drag suppression for 2D oscillating cylinder with various arrangement of splitters at $\operatorname{Re}=100$ : a high-amplitude study with OpenFOAM," Journal of Wind Engineering \& Industrial Aerodynamics, vol. 164, pp. 128-137, 2017.

[5] Y. Qiu, Y. Sun, Y. Wu, and Y. Tamura, "Effects of splitter plates and Reynolds number on the aerodynamic loads acting on a circular cylinder," Journal of Wind Engineering \& Industrial Aerodynamics, vol. 127, pp. 40-50, 2014.

[6] M. Lou, Z. Chen, and P. Chen, "Experimental investigation of the suppression of vortex induced vibration of two interfering risers with splitter plates," Journal of Natural Gas Science and Engineering, vol. 35, pp. 736-752, 2016.

[7] G. R. S. Assi, P. W. Bearman, and N. Kitney, "Low drag solutions for suppressing vortex-induced vibration of circular cylinders," Journal of Fluids and Structures, vol. 25, no. 4, pp. 666-675, 2009.

[8] G. R. S. Assi, P. W. Bearman, and M. A. Tognarelli, "On the stability of a free-to-rotate short-tail fairing and a splitter plate as suppressors of vortex-induced vibration," Ocean Engineering, vol. 92, pp. 234-244, 2014.

[9] B. Gozmen, H. Akilli, and B. Sahin, "Passive control of circular cylinder wake in shallow flow," Measurement, vol. 46, no. 3, pp. 1125-1136, 2013.

[10] H. Zhu, Y. Lin, Q. Jia, and X. Yang, "Simulations of suppressive effect of VIV on marine riser with splitter plates," in Proceedings of the 3rd IEEE International Conference on Computer Science and Information Technology (IACSIT '10), vol. 6, p. 5, IEEE 
China Council, IEEE Beijing Section Sichuan Computer Federation, 2010.

[11] F. Gu, J. S. Wang, X. Q. Qiao, and Z. Huang, "Pressure distribution, fluctuating forces and vortex shedding behavior of circular cylinder with rotatable splitter plates," Journal of Fluids and Structures, vol. 28, pp. 263-278, 2012.

[12] H. Akilli, B. Sahin, and N. F. Tumen, "Suppression of vortex shedding of circular cylinder in shallow water by a splitter plate," Flow Measurement and Instrumentation, vol. 16, no. 4, pp. 211219, 2005.

[13] Y. Z. Law and R. K. Jaiman, "Wake stabilization mechanism of low-drag suppression devices for vortex-induced vibration," Journal of Fluids and Structures, vol. 70, pp. 428-449, 2017.

[14] G. R. S. Assi and P. W. Bearman, "Transverse galloping of circular cylinders fitted with solid and slotted splitter plates," Journal of Fluids and Structures, vol. 54, pp. 263-280, 2015.

[15] L. Ma, S. Hu, M. Qiu, Q. Li, and Z. Ji, "Energy consumption optimization of high sulfur natural gas purification plant based on back propagation neural network and genetic algorithms," Energy Procedia, vol. 105, pp. 5166-5171, 2017.

[16] W.-Z. Jiang, S.-Q. Duan, C.-M. Yang et al., "Optimal design of centrifugal pump impeller based on CFD simulations and BP neural network," Machine Tool \& Hydraulic, vol. 44, no. 22, pp. 67-70, 2016.

[17] C.-J. Liang, G.-L. Yang, X.-F. Wang et al., "Dynamic optimization of artillery structure based on neural network and genetic algorithm," Journal of Ordnance Industry, vol. 36, no. 5, pp. 789794, 2015.

[18] A. M. C. Smith, C. Yang, H. Ma, P. Culverhouse, A. Cangelosi, and E. Burdet, "Novel hybrid adaptive controller for manipulation in complex perturbation environments," PLoS ONE, vol. 10, no. 6, Article ID e0129281, 2015.

[19] C. Yang, Z. Li, and J. Li, “Trajectory planning and optimized adaptive control for a class of wheeled inverted pendulum vehicle models," IEEE Transactions on Cybernetics, vol. 43, no. 1, pp. 24-36, 2013.

[20] H. Safikhani, A. Abbassi, A. Khalkhali, and M. Kalteh, "Multiobjective optimization of nanofluid flow in flat tubes using CFD, Artificial Neural Networks and genetic algorithms," Advanced Powder Technology, vol. 25, no. 5, pp. 1608-1617, 2014.

[21] H. Avci, D. Kumlutaş, Ö. Özer, and M. Özşen, “Optimisation of the design parameters of a domestic refrigerator using CFD and artificial neural networks," International Journal of Refrigeration, vol. 67, pp. 227-238, 2016.

[22] W. Tian, Z. Mao, and F. Zhao, "Design and numerical simulations of a flow induced vibration energy converter for underwater mooring platforms," Energies, vol. 10, no. 9, p. 1427, 2017.

[23] W. Tian, Z. Mao, X. An, B. Zhang, and H. Wen, "Numerical study of energy recovery from the wakes of moving vehicles on highways by using a vertical axis wind turbine," Energy, vol. 141, pp. 715-728, 2017.

[24] ANSYS Inc, Fluent 15.0 User's Guide, 2016.

[25] C. Yang, Z. Li, R. Cui, and B. Xu, "Neural network-based motion control of an underactuated wheeled inverted pendulum model," IEEE Transactions on Neural Networks and Learning Systems, vol. 25, no. 11, pp. 2004-2016, 2014.

[26] X. Wang, C. Yang, Z. Ju et al., "Robot manipulator self-identification for surrounding obstacle detection," Multimedia Tools \& Applications, vol. 76, pp. 6495-6520, 2017.

[27] E. F. Kreith, M. J. Moran, and G. Tsatsaronis, "Engineering thermodynamics," in The CRC Handbook of Thermal Engineering, F. Kreith, Ed., CRC Press LLC, Boca Raton, Fla, USA, 2000.
[28] B. Wang, Heat Transfer Science and Technology 2000, Higher Education Press, 2000.

[29] X.-C. Wang, There Are 43 Case Studies of MATLAB Neural Network, Beijing University of Aeronautics and Astronautics Press, 2013.

[30] C. H. K. Williamson and A. Roshko, "Vortex formation in the wake of an oscillating cylinder," Journal of Fluids and Structures, vol. 2, no. 4, pp. 355-381, 1988. 


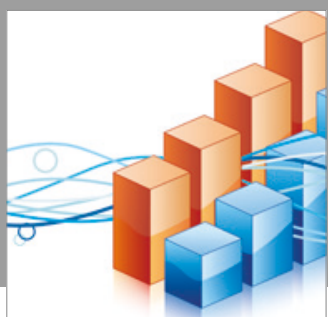

Advances in

Operations Research

vatersals

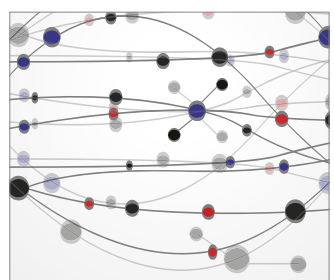

\section{The Scientific} World Journal
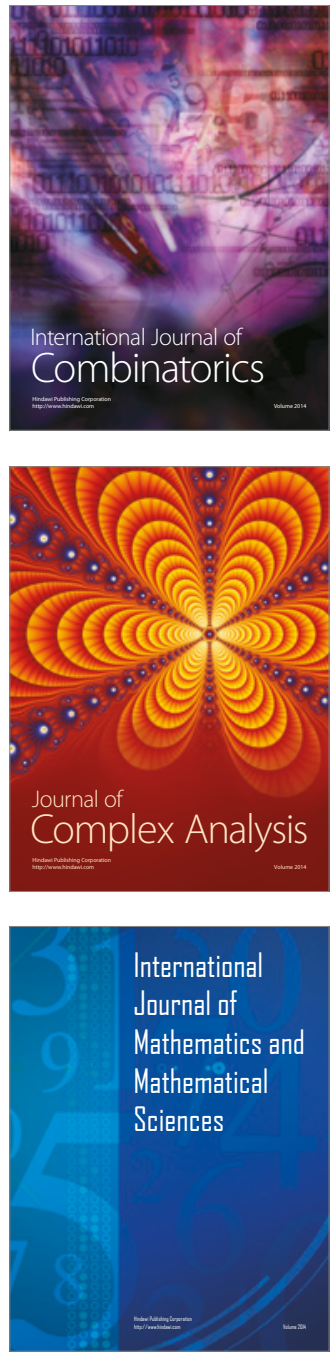
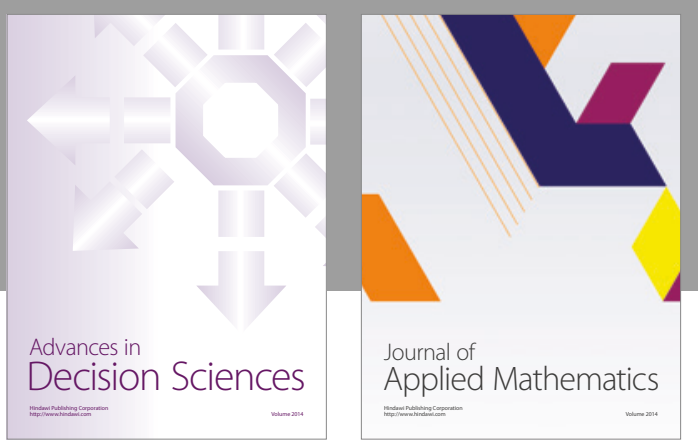

Algebra

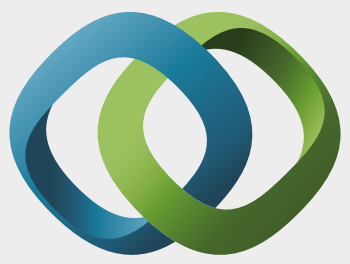

\section{Hindawi}

Submit your manuscripts at

https://www.hindawi.com
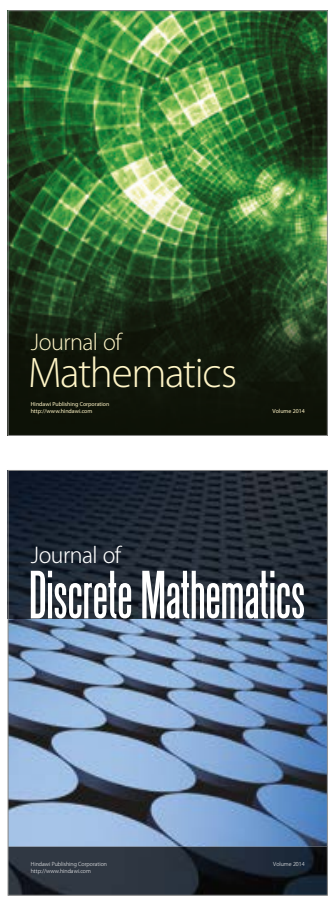

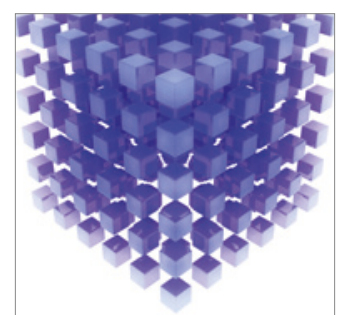

Mathematical Problems in Engineering
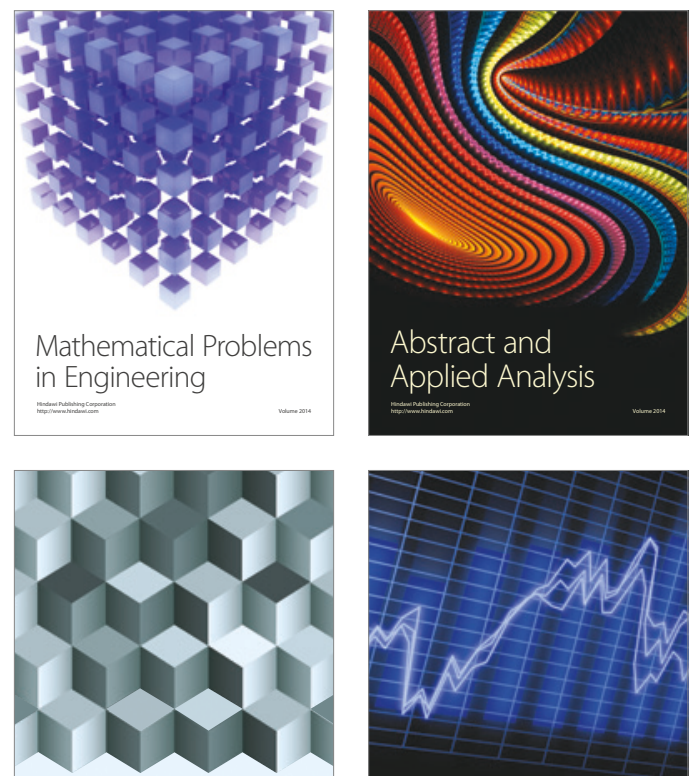

Journal of

Function Spaces

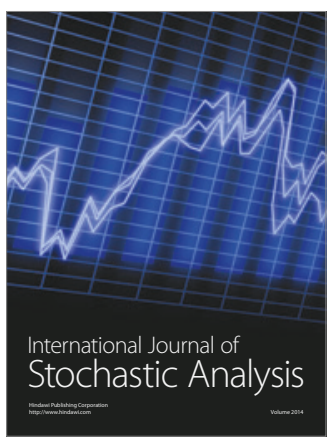

Probability and Statistics
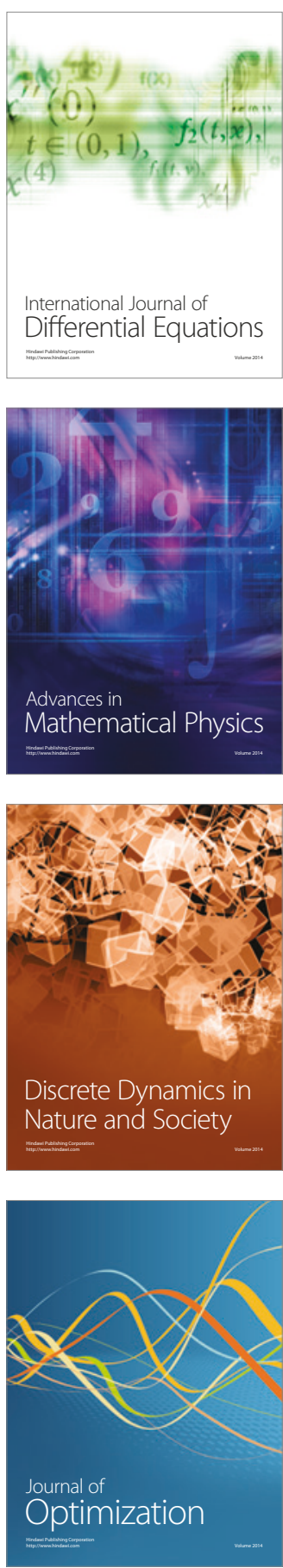